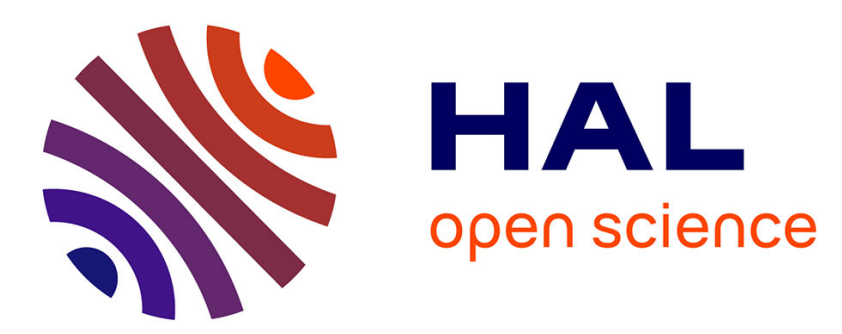

\title{
A review of the use and potential of the GATE Monte Carlo simulation code for radiation therapy and dosimetry applications
}

D. Sarrut, M. Bardiès, N. Boussion, N. Freud, S. Jan, Jean Michel Létang, G. Loudos, L. Maigne, S. Marcatili, T. Mauxion, et al.

\section{To cite this version:}

D. Sarrut, M. Bardiès, N. Boussion, N. Freud, S. Jan, et al.. A review of the use and potential of the GATE Monte Carlo simulation code for radiation therapy and dosimetry applications. Medical Physics, 2014, 41, pp.064301. 10.1118/1.4871617 . hal-01015819

\author{
HAL Id: hal-01015819 \\ https://hal.science/hal-01015819
}

Submitted on 20 Nov 2018

HAL is a multi-disciplinary open access archive for the deposit and dissemination of scientific research documents, whether they are published or not. The documents may come from teaching and research institutions in France or abroad, or from public or private research centers.
L'archive ouverte pluridisciplinaire HAL, est destinée au dépôt et à la diffusion de documents scientifiques de niveau recherche, publiés ou non, émanant des établissements d'enseignement et de recherche français ou étrangers, des laboratoires publics ou privés. 


\section{EXPECT NOTHING LESS THAN SUB-MILLIMETER ACCURACY IN YOUR MEASUREMENTS}

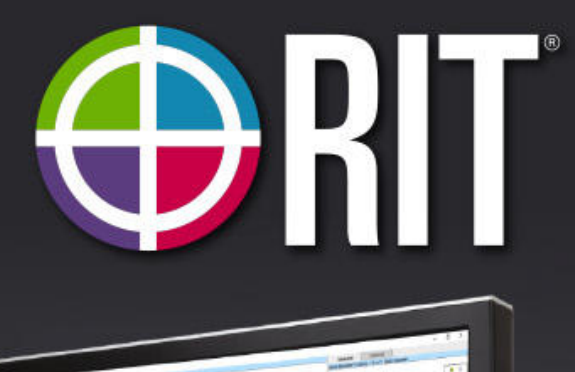

\section{START UTILIZING ADVANCED PRECISION QA TODAY}

\section{THE RIT FAMILY OF PRODUCTS VERSION 6.7.X}

\section{D Winston-Lutz Isocenter Optimization}

The enhanced isocenter optimization routine allows for optimal cone detection, resulting in the utmost precision. The accuracy of this routine now surpasses sub-millimeter resolution. (Pictured above)

\section{Cerberus 2.0: The Future of Complete Automation} Completely streamline your automated phantom analysis workflow with the new Cerberus. Cerberus operates in the background of your workstation, allowing for hands-free Imaging $\mathrm{QA}$.

\section{One-Click, Instant Automated Phantom Analyses}

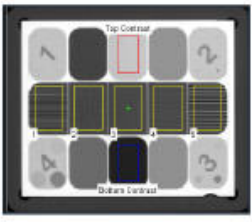

RIT's full suite of phantom

analyses provides fast, robust, and accurate analysis of all imaging tests recommended in TG-142. The software now offers added support for the QCkV-1 phantom. (Pictured right)

Tolerance Customization and Management RIT's new Tolerance Manager offers comprehensive customization of tolerance values for every measurement used in all automated phantom analyses. Tolerance profiles can be precisely-tailored to each individual machine in use.
Elekta Leaf Speed Analysis

RIT now offers fast and accurate automated analysis for Elekta MLC QA. The new Leaf Speed Analysis routine measures the consistency and accuracy of the MLC leaf speeds as they traverse the imager.

\section{TomoTherapy ${ }^{\circledast}$ Registration for Patient QA}

Easily perform exact dose comparisons with RIT's new

TomoTherapy Registration routine. This new, innovative wizard uses a TomoTherapy ${ }^{\circledR}$ plan, dose map, and a film to determine position an dose accuracy using the red lasers. (Pictured below)

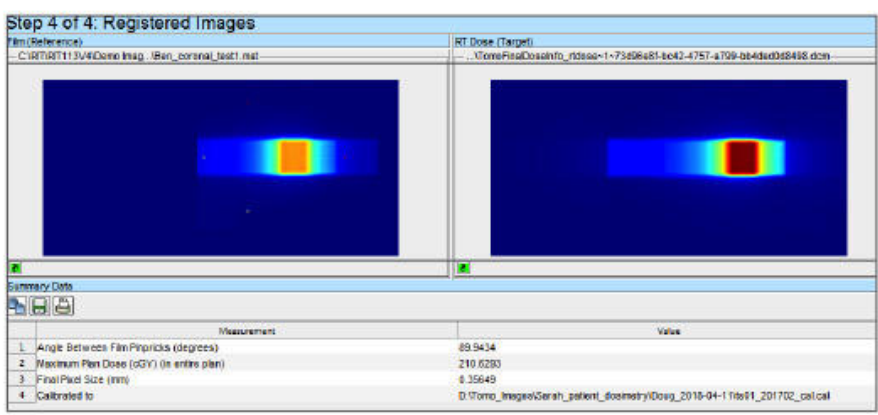

Streamline Your QA Workflow

Fully-customize your software experience with RIT's updated and dynamic interface. With the new ability to hide/display any features or sections, you can instantly access you most frequently-performed analysis routines.

\section{Convenient, Cloud-Based Software Licensing}

Easily manage your software licenses with RIT's new, flexible system at your convenience, 24/7/365 without the help of RIT Technical Support. Upgrading is now easier than ever. 


\title{
A review of the use and potential of the GATE Monte Carlo simulation code for radiation therapy and dosimetry applications
}

\author{
David Sarrut $\left.{ }^{a}\right)$ \\ Université de Lyon, CREATIS; CNRS UMR5220; Inserm U1044; INSA-Lyon; Université Lyon 1; \\ Centre Léon Bérard, France \\ Manuel Bardiès \\ Inserm, UMR1037 CRCT, F-31000 Toulouse, France and Université Toulouse III-Paul Sabatier, \\ UMR1037 CRCT, F-31000 Toulouse, France \\ Nicolas Boussion \\ INSERM, UMR 1101, LaTIM, CHU Morvan, 29609 Brest, France \\ Nicolas Freud \\ Université de Lyon, CREATIS, CNRS UMR5220, Inserm U1044, INSA-Lyon, Université Lyon 1, \\ Centre Léon Bérard, 69008 Lyon, France
}

Sébastien Jan

CEA/DSV/I2BM/SHFJ, Orsay 91401, France

Jean-Michel Létang

Université de Lyon, CREATIS, CNRS UMR5220, Inserm U1044, INSA-Lyon, Université Lyon 1, Centre Léon Bérard, 69008 Lyon, France

George Loudos

Department of Medical Instruments Technology, Technological Educational Institute of Athens, Athens 12210, Greece

Lydia Maigne

UMR 6533 CNRS/IN2P3, Université Blaise Pascal, 63171 Aubière, France

Sara Marcatili and Thibault Mauxion

Inserm, UMR1037 CRCT, F-31000 Toulouse, France and Université Toulouse III-Paul Sabatier, UMR1037 CRCT, F-31000 Toulouse, France

Panagiotis Papadimitroulas

Department of Biomedical Engineering, Technological Educational Institute of Athens, 12210, Athens, Greece

Yann Perrot

UMR 6533 CNRS/IN2P3, Université Blaise Pascal, 63171 Aubière, France

Uwe Pietrzyk

Institut für Neurowissenschaften und Medizin, Forschungszentrum Jülich GmbH, 52425 Jülich, Germany and Fachbereich für Mathematik und Naturwissenschaften, Bergische Universität Wuppertal, 42097 Wuppertal, Germany

Charlotte Robert

IMNC, UMR 8165 CNRS, Universités Paris 7 et Paris 11, Orsay 91406, France

Dennis R. Schaart

Delft University of Technology, Faculty of Applied Sciences, Radiation Science and Technology Department, Delft Mekelweg 15, 2629 JB Delft, The Netherlands

Dimitris Visvikis

INSERM U1101, LaTIM, CHU Morvan, 29609 Brest, France

Irène Buvat

IMNC, UMR 8165 CNRS, Universités Paris 7 et Paris 11, 91406 Orsay, France and CEA/DSV/I2BM/SHFJ, 91400 Orsay, France

(Received 16 September 2013; revised 24 March 2014; accepted for publication 30 March 2014; published 12 May 2014)

In this paper, the authors' review the applicability of the open-source GATE Monte Carlo simulation platform based on the GEANT4 toolkit for radiation therapy and dosimetry applications. The many applications of GATE for state-of-the-art radiotherapy simulations are described including external beam radiotherapy, brachytherapy, intraoperative radiotherapy, hadrontherapy, molecular radiotherapy, and in vivo dose monitoring. Investigations that have been performed using GEANT4 only are also mentioned to illustrate the potential of GATE. The very practical feature of GATE making it easy to model both a treatment and an imaging acquisition within the same framework 
is emphasized. The computational times associated with several applications are provided to illustrate the practical feasibility of the simulations using current computing facilities. (C) 2014 American Association of Physicists in Medicine. [http://dx.doi.org/10.1118/1.4871617]

Key words: Monte-Carlo simulation, dosimetry, radiation therapy

\section{INTRODUCTION}

Monte Carlo (MC) simulation is widely recognized as an essential method to study the physics of nuclear medicine, radiology, and radiation therapy. The concepts of deposited energy and absorbed dose are of particular interest for radiotherapy applications ${ }^{1}$ and imaging applications involving ionizing radiations. ${ }^{2}$ In radiation therapy (RT), treatment planning requires an accurate assessment of the absorbed dose distribution throughout the organs and tissues of interest. This is true for a large variety of RT approaches (e.g., using photons, electrons, protons, carbon beams, radioisotopes) with different delivery conditions (broad beam, pencil beam, scanning, rotational, brachytherapy, and targeted radionuclide therapy). In diagnostic imaging applications involving ionizing radiation, such as computed tomography $(\mathrm{CT})$, positron emission tomography (PET), or single photon emission tomography (SPECT), the assessment of the absorbed dose is important to better analyze the risk-benefit of the procedure. Imaging and therapy are increasingly tied together: cone-beam or portal imaging are associated with conventional linac RT, CT acquisitions are performed during tomotherapy, radiographs pairs are acquired during Cyberknife treatments, and new imaging systems are developed for treatment monitoring in hadrontherapy, such as hadron-PET, ${ }^{3,4}$ promptgamma (PG) cameras, ${ }^{5,6}$ and interaction vertex imaging (IVI) systems. ${ }^{7}$ As a result, there is a need for a MC simulation platform supporting radiation transport modeling for (combined) imaging and dosimetry applications.

Many MC simulation tools have been developed for imaging (e.g., Refs. 8-10) or dosimetry (e.g., Refs. 11-16). At the moment, GATE (Refs. 17 and 18) is the only open-source MC simulation platform supporting the user-friendly simulation of imaging, RT and dosimetry in the same environment. GATE is an application based on the GEANT4 toolkit: GEANT4 manages the kernel that simulates the interactions between particles and matter, and GATE provides additional high-level features to facilitate the design of GEANT4based simulations. GATE is developed by the OpenGate collaboration ${ }^{19}$ and is a community-driven initiative, where every user can access the source code ${ }^{20}$ and propose new features.

GATE is potentially useful for a broad range of simulations, including those where the absorbed dose is the principal observable. While GATE has been widely validated and used for a large variety of PET and SPECT studies, there are still a limited number of papers reporting its application and reliability in the context of dosimetry. The purpose of this paper is therefore to review the current status of GATE for dosimetry-related applications based on published validation works. Source macros for five applications discussed here (brachytherapy, external beam radiotherapy with photons/ electrons, molecular radiotherapy, and protontherapy) are provided as additional material for the interested readers. As GATE is based on GEANT4, validated GEANT4 applications related to dosimetry are mentioned as application fields where GATE could be successfully used. Upcoming developments in GATE regarding RT and dosimetry applications are also discussed.

\section{ABSORBED DOSE CALCULATION IN GATE}

In this paper, we focus on the absorbed dose $D$, defined as the deposited energy per unit mass of medium, reported in units of gray $(1 \mathrm{~Gy}=1 \mathrm{~J} / \mathrm{kg})$. In MC simulations, the energy deposited in a volume Edep is usually expressed in $\mathrm{eV}\left(1 \mathrm{eV}=1.60217646 \times 10^{-19} \mathrm{~J}\right)$. It can be converted to Gy by accounting for the volume of interest and the density: $D[\mathrm{~Gy}]=\left(E_{d e p}[\mathrm{eV}] \times 1.60217646\right.$ $\left.\times 10^{-19}[\mathrm{~J} / \mathrm{eV}]\right) /\left(\rho\left[\mathrm{kg} / \mathrm{cm}^{3}\right] / V\left[\mathrm{~cm}^{3}\right]\right)$.

The absorbed dose $D$ is a physical quantity and does not reflect the biological effects of irradiation. However, $D$ is the first step toward the assessment of the biological impact of radiation, both for stochastic and deterministic effects. GATE is provided with a mechanism, named DoseActor, which stores the absorbed dose in a given volume in a 3D matrix. In terms of GATE macros, the dose actor has to be attached to the volume of interest. The user can provide the matrix size and the matrix position is defined within the coordinate system of the monitored volume. Note that if the user defines a matrix size larger than the attached volume, the absorbed dose deposition occurring outside the volume but inside the matrix will not be recorded. The actor calculates the deposited energy in $\mathrm{MeV}\left(E_{\mathrm{dep}}\right)$, the absorbed dose $D$ in Gy, the number of hits (a "hit" occurs each time a primary or secondary particle makes a step in a volume, with or without energy deposition), and the local statistical uncertainty according to Ref. 21. The squared sums of $E_{\text {dep }}$ and $D$ are also provided and can be used to compute the statistical uncertainty when the simulation is split into multiple jobs. Equation (1) defines the statistical uncertainty $\varepsilon_{k}$ at pixel $k$, with $N$ being the number of primary events, $d_{k, i}$ the deposited energy in pixel $k$ at primary event $i$. The absorbed dose can be calculated as dose-to-water, as traditionally performed in RT, and as dose-to-medium as in conventional MC simulations. ${ }^{22,23}$ The conversion is performed on the fly by accounting for the relative stopping power and the energy transferred via nuclear interactions in the specific medium. Note that this conversion method may not be appropriate for some situations in brachytherapy ${ }^{24}$

$$
\begin{aligned}
& D_{k}=\sum_{i}^{N} d_{k, i} \quad S_{k}=\sqrt{\frac{1}{N-1}\left(\frac{\sum_{i}^{n} d_{k, i}^{2}}{N}-\left(\frac{\sum_{i}^{n} d_{k, i}}{N}\right)^{2}\right)} \\
& \varepsilon_{k}=100 \times \frac{S_{k}}{D_{k}} .
\end{aligned}
$$


During particle tracking, the deposited energy is summed in the matrix for each step occurring in the attached volume. The two endpoints of a step are called the PreStep point and PostStep point. For a charged particle, a position is randomly chosen along the step and the values are stored in the matrix at that position. For a photon, when secondary particles are not created because the energy is below the production threshold, energy is deposited at the PostStep position. The user has to make sure that the step length is not too large with respect to the matrix sampling. The output can be stored in mhd image file format, composed of a header file and a raw data file. This file format can be handled by several opensource image processing toolkits, such as ITK (www.itk.org) and ImageJ (rsbweb.nih.gov/ij). The coordinate system of the image (called "origin") within the scene (named the "world") is recorded. It therefore allows for positioning the absorbed dose matrix in relation to the attached volume for visualisation purposes. Other file formats, such as $h d r$ (Analyze), $t x t$, and root (http://root.cern.ch), are also supported.

The insertion of CT data into GEANT4 was described in Ref. 25. The influence of the calibration of the Hounsfield Units (HU) into material and density has also been reported. ${ }^{26}$ In GATE, the user can manually assign a material to any HU range or use the Schneider method ${ }^{27}$ based on a predefined group of materials ( 24 by default). Two mixtures with the same elemental composition but different densities are considered as two distinct materials. Each requires the computation of cross-section, stopping power, and other tables, which can be a problem if too many materials are used. In Ref. 25, the authors described a method to dynamically change the density at run time but this technique is not yet available in GATE. In GATE, the number of materials can be controlled with the density tolerance parameter. ${ }^{28}$ It is used to split a material into two materials when its corresponding $\mathrm{HU}$ range is larger than the tolerance.

Variance reduction techniques (VRT) and the use of cuts to speed up simulations are available within GATE. Production cuts can be set for preventing secondary photons, electrons, positrons, or protons to be generated if their energy is below a user-defined threshold. Cuts are expressed in distance, thus depending on the material, or in energy. Cuts can be set to different values in different regions. Regarding VRT, splitting and Russian roulette methods are available. User can choose to use VRT only in selected situations by employing "filters." For example, selective bremsstrahlung splitting (SBS) is described in Ref. 29. For photon tracking without modeling dose deposits, fast fictitious interaction tracking is available. ${ }^{30}$ Particle tracking in voxelized geometry can also be accelerated with region-oriented CT representation. ${ }^{28}$ Since the GATE V6.2 release, a specific VRT option is provided to accelerate absorbed dose calculations for low energy photons (from $1 \mathrm{keV}$ to a few hundred $\mathrm{keV}$ ) in the kerma approximation. ${ }^{31}$ This method is based on the track length estimator (TLE) ${ }^{32}$ Efficiency gains between 10 and $10^{3}$ can be obtained depending on the simulated configuration. Two additional low energy photon VRT, force-detection, ${ }^{33}$ and exponential TLE, ${ }^{34}$ are currently being developed and will be available in future GATE releases.
Finally, a GPU (graphical processing units) option of the DoseActor is being developed by the OpenGATE collaboration. ${ }^{35}$ With this option, particles within the attached volume are no longer tracked by GEANT4 but by a specific GPU process, highly reducing the simulation time. Although there are currently certain limitations in the GPU implementation (photon and electron processes only, no observables during the GPU tracking process), there is a significant impact in the overall resulting computational efficiency. This GPU option will be integrated within the GATE V7 version supporting combined CPU and GPU calculations in various applications.

\section{APPLICATIONS}

In this section, we present several types of dosimetric applications of GATE: molecular radiotherapy (MRT), brachytherapy, intraoperative radiotherapy (IORT), external beam RT (EBRT), particle therapy, and in vivo absorbed dose monitoring. We also mention dose-related applications that have only been addressed with GEANT4 so far, but where GATE, being based on the GEANT4 toolbox, could be potentially used with success.

\section{A. Molecular radiotherapy}

\section{A.1. Dose point kernel}

The use of MC in targeted radionuclide therapy or molecular radiotherapy has been first dedicated to the simulation of dose point kernels (DPK), i.e., the radial deposition of energy around a point source in a homogeneous medium, ${ }^{36}$ for example, with EGS. ${ }^{37}$ Any radioactive volume can be considered as a juxtaposition of independent point sources (superposition principle). Therefore, in a homogenous medium, the variation of absorbed dose with the distance from a point source is sufficient to obtain, by convolution, the absorbed dose for any radioactive distribution. This is the rationale for the computation of DPKs that are used intensively in radiopharmaceutical dosimetry. ${ }^{38}$ Interestingly, the simple assumptions (point source, usually monoenergetic particles, energy scoring in spherical shells, homogeneous material) make DPK results an interesting benchmark for MC codes. ${ }^{39-41}$

An early application of GATE for electron DPKs is described in Ref. 42. More recently, in Ref. 43 the authors studied the energy deposition for mono-energetic electrons using GATE V6. Comparisons with EGSnrc and MCNP4C showed good agreement for electrons with energies between $15 \mathrm{keV}$ and $4 \mathrm{MeV}$, as long as parameters were correctly set, a point consistent with earlier observations. ${ }^{44}$ At the moment, DPKbased algorithms are based on the assumption that the human body is equivalent to water, without taking into account tissue variations. The combination of DPKs with patient SPECT or PET data can provide a fast absorbed dose calculation, which takes into account patient specific anatomic information. A fast algorithm was proposed in Ref. 45. GATE V6.1 has been used for extending the existing DPKs in more tissues and for a variety of nuclear radio-isotopes. Specifically 
in Ref. 46, DPKs were generated using GATE V6.1 for $10 \mathrm{keV}$ to $10 \mathrm{MeV}$ electrons and various radionuclides $\left({ }^{177} \mathrm{Lu}\right.$, ${ }^{90} \mathrm{Y},{ }^{32} \mathrm{P},{ }^{111} \mathrm{In},{ }^{131} \mathrm{I},{ }^{125} \mathrm{I}$, and ${ }^{99 \mathrm{~m}} \mathrm{Tc}$ ), for water, bone, and lungs. A comparison between results and data from the literature obtained using different codes (MCNP, EGS, FLUKA, ETRAN, GEPTS, and PENELOPE) showed a general agreement within $6 \%$. An additional DPK dataset was then generated for other radionuclides of interest $\left({ }^{67} \mathrm{Ga},{ }^{68} \mathrm{Ga},{ }^{123} \mathrm{I},{ }^{124} \mathrm{I}\right.$, ${ }^{125} \mathrm{I},{ }^{153} \mathrm{Sm},{ }^{186} \mathrm{Re}$, and $\left.{ }^{188} \mathrm{Re}\right)$. The conclusion is that current MC codes-including GATE—now provide equivalent results for "standard" situations, involving photon/electron transport for energies of interest in nuclear medicine. The application of DPKs in voxel-based geometries can be found in the computation of "Dose Voxel Kernels" or "Voxel S Values," i.e., input data suited for a convolution with voxel-based activity estimate as obtained in digital imaging. Some work using GEANT4 was also recently reported by Amato et al. ${ }^{47}$ with $3 \mathrm{D}$ arrays of $11 \times 11 \times 11$ cubic voxels, $3 \mathrm{~mm}$ in size, for several emitters of interest in nuclear medicine $\left({ }^{32} \mathrm{P},{ }^{90} \mathrm{Y}\right.$, ${ }^{99 \mathrm{~m}} \mathrm{Tc},{ }^{177} \mathrm{Lu},{ }^{131} \mathrm{I},{ }^{153} \mathrm{Sm},{ }^{186} \mathrm{Re},{ }^{188} \mathrm{Re}$ ), further demonstrating the potential of GATE for that kind of applications.

Alpha particle and low energy (Auger) electron targeted therapy corresponds to the specific and challenging situation of cellular radiopharmaceutical dosimetry. GATE has not been used for such applications so far, but GEANT4 has, ${ }^{48,49}$ so GATE could support such applications. Moreover, another GEANT4-based tool, GEANT4-DNA ${ }^{50}$ is being developed to deal with very low energy radiation transport at the molecular level..$^{51}$

\section{A.2. $S$ values and absorbed dose calculations}

MC codes are used to compute reference values for dosimetry, most often in terms of the "mean absorbed dose per decay," i.e., the $\mathrm{S}$ value, or Dose Conversion Factor. ${ }^{52,53}$ These values are then used to derive reference absorbed dose values for nuclear medicine practice (ICRP 1987, 2008). This relies on the acceptance of reference geometries described as anthropomorphic phantoms [ICRP 2009 (Ref. 54)]. The same approach can be used for preclinical experiments where rodent models have been proposed for radiopharmaceutical dosimetry. ${ }^{55}$ Alternatively, direct absorbed dose calculation can be performed for a specific geometry/radiopharmaceutical distribution dataset. This is the basis of patient-specific dosimetry.

Preclinical dosimetry. GATE has been used for preclinical dosimetry ${ }^{56}$ to study the energy deposition from ${ }^{18} \mathrm{~F}$ labeled radiopharmaceuticals in mice. Four models were considered: a voxelized MOBY, ${ }^{57}$ using a $400 \mu \mathrm{m}$ spatial sampling, and three high-resolution models of bladder, femur, and vertebra, with a spatial sampling of 50,15 , and $25 \mu \mathrm{m}$, respectively. Absorbed dose per injected activity (mGy/MBq) was computed for the various organs of interest for FDG, FLT, and fluoride. Absorbed dose volume histograms were generated.

Using GEANT4 only, Keenan et al. ${ }^{55}$ assessed the impact of various parameters (material definition/densities, fine suborgan segmentation for airways, bones, heart, and stomach) on ${ }^{18} \mathrm{~F} \mathrm{~S}$ values.
A comparison of their results, ${ }^{18} \mathrm{~F} \mathrm{~S}$ values obtained for the MOBY 30g Nude Mouse model with MCNPX 2.7a and GATE V6.1, yielded very close results, with most relative differences in $\mathrm{S}$ values below $5 \%{ }^{58} \mathrm{~S}$ value computation for positron emitting radionuclides with MOBY2 phantom (mouse/rat) using GATE V6.1 is ongoing. ${ }^{59,60}$

Clinical dosimetry. Aside from an early presentation in $2006,{ }^{61}$ clinical nuclear medicine dosimetry results using GATE are recent. In Ref. 62, GATE was used to compute specific absorbed fractions (SAF) for photon sources in selected organs of a reference anthropomorphic model. The Snyder mathematical model ${ }^{63}$ was digitized and sampled (2 $\times 2 \times 3 \mathrm{~mm})$ to generate GATE input files for photons $(10 \mathrm{keV}$ to $1 \mathrm{MeV})$. Six sources of interest were considered: kidneys, liver, lungs, pancreas, spleen, and adrenals. The results for self-irradiation are very close to values published in Ref. 63, except for energies below $30 \mathrm{keV}$. The same trend can be observed for cross-irradiation. It must be stressed that early results published for the Snyder phantom were obtained with simplifying assumptions and radiation transport was addressed with a methodology that has been markedly improved since. Therefore, it is not clear if values presented in Ref. 63 should still be considered as a reference.

GATE calculations ( $\mathrm{S}$ values and SAFs) performed on the Zubal phantom ( $4 \times 4 \times 4 \mathrm{~mm}$ sampling) have been compared with MCNP-4B and MCNPX published data. ${ }^{64-66} \mathrm{Al}-$ though fairly good agreement between the various datasets was reported, some discrepancies were observed, especially for very low $\mathrm{S}$ values where the associated statistical uncertainty was high. In a further study, the relevance of processing paired organs as a single entity for absorbed dose calculation was investigated. This work was performed using the Zubal phantom ${ }^{67}$ and GATE V6 and concluded that paired organs should be treated separately for absorbed dose calculations. Other GATE-based dosimetry work using the Zubal phantom ${ }^{68}$ demonstrated the importance of accounting for the dose volume histogram in addition to the mean and maximum absorbed dose when investigating the dose-response relationship in ${ }^{131}$ I-targeted radiotherapy. Another study ${ }^{69}$ compared the results obtained on six healthy volunteers during a clinical study evaluating a new ${ }^{18} \mathrm{~F}$-labeled PET/CT brain tracer. A patient-specific approach was employed using MCNPX 2.5 and GATE V6.1. The validation of MC calculations by comparing GATE and MCNPX results versus OLINDA $S$ values for ${ }^{18} \mathrm{~F}$ on a voxelized MIRD phantom was successful with less than $8 \%$ relative difference for mean organ self-absorbed doses. Figure 1 illustrates an absorbed dose distribution computed with GATE in a mouse from a ${ }^{131} \mathrm{I}$ source overlaid on the mouse CT.

\section{B. Brachytherapy}

\section{B.1. Context}

Since the 1990s, MC simulations have played an increasing role in the characterization of brachytherapy devices. ${ }^{70} \mathrm{~A}$ dosimetry formalism for photon brachytherapy sources has been proposed in 1995, revised in 2004 by the AAPM 


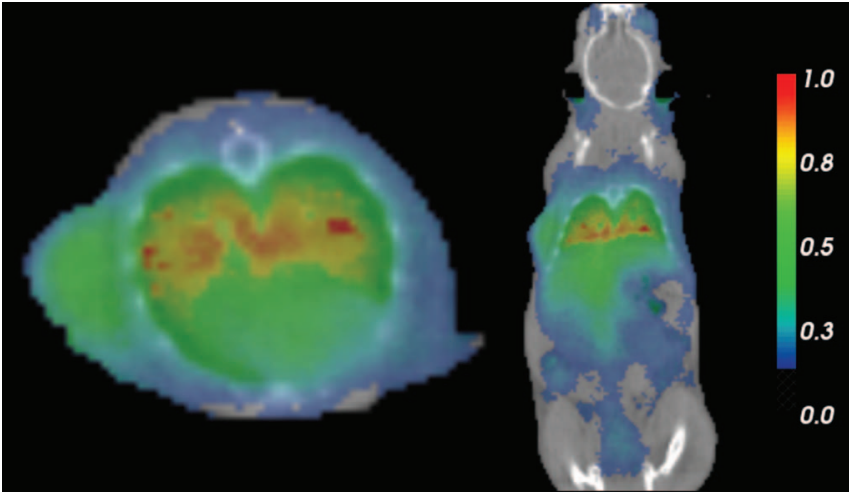

FIG. 1. Dose distribution (normalized to the maximum dose) overlaid on a mouse CT, obtained from an ${ }^{131}$ I source.

Radiation Therapy Committee Task Group No. 43 (Ref. 71) and updated again in No. $186 .{ }^{72}$ MC simulations were used to calculate the dosimetry parameters such as the air kerma strength, radial absorbed dose function, anisotropy function and absorbed dose rate constant in liquid water. These simulations were validated by comparison with measurements, and consensus datasets were proposed for incorporation into brachytherapy treatment planning systems (TPS). Some MC studies have demonstrated limitations of the TG-43 formalism, ${ }^{73,74}$ e.g., due to the effects of source shielding, interseed attenuation, and tissue heterogeneity. TG-186 provided guidance on the usage of model-based dose calculation algorithms (MBDCAs), such as collapsed-cone (CC) convolution, superposition convolution, MC methods, and more recently grid-based Boltzmann solver (GBBS) to simulate radiation transport in nonwater media. For beta particle brachytherapy sources, the AAPM TG-60 formalism is generally recommended to calculate the dosimetry parameters. ${ }^{75,76}$

\section{B.2. GATE and GEANT4 studies}

The only GATE application for brachytherapy involving gamma sources has been reported by Thiam et al. ${ }^{77}$ who explored low energy ${ }^{125} \mathrm{I}$ source dosimetry. The reported results agree with consensus values with a relative accuracy better than $2 \%$, suggesting the validity of GATE for such applications. Yet, the potential of GATE for brachytherapy application is mostly demonstrated by several studies regarding brachytherapy performed with the GEANT4 toolkit. Figure 2 shows an example of a dose distribution for a low dose rate brachytherapy treatment using $79^{125}$ I seeds.

Electromagnetic physics processes of GEANT4 at low energy (around few MeV) have been evaluated for brachytherapy in several publications. Several electromagnetic packages (standard, low energy, PENELOPE) and numerous parameters are available and recommendations have been provided. Some of those packages underwent changes between versions. To assist users, GEANT4 and GATE integrate predefined physics lists, i.e., preformatted setting of the physics models and associated parameters for dosimetry purposes.

Among the GEANT4 studies, Granero et al. ${ }^{78}$ compared GEANT4 to the Plaque Simulator TPS for ocular brachytherapy treatment using eye plaques loaded with ${ }^{125} \mathrm{I}$ radioactive sources. They showed the lack of accuracy of the TPS around the border of the eye. Pérez-Calatayud et al. ${ }^{79,80}$ demonstrated that the standard electromagnetic package of GEANT4 coupled with the low energy package for the Rayleigh scattering gave reliable results for ${ }^{137} \mathrm{Cs}$ sources. All these packages are available in GATE and could thus be used for similar simulations. Meigooni et al. ${ }^{81}$ obtained 5\% agreement between measured and simulated radial absorbed dose and anisotropy functions. GEANT4 was also used to model ${ }^{192}$ Ir sources. ${ }^{79,82-85}$ Other dosimetric studies regarding brachytherapy applicators in water have been performed with GEANT4 (Refs. 86-89) and new candidates for treatments have also been investigated, such as ${ }^{57} \mathrm{Co}$ or ${ }^{170} \mathrm{Tm} \cdot{ }^{90,91}$ In Ref. 92, GEANT4 was used to characterize the x-ray source of an electronic brachytherapy system and comparisons with BEAMnrc simulations were reported. HVLs (half-value layers) and attenuation measurements obtained with the two codes mostly agreed within uncertainty limits, while discrepancies between simulations and measurements were observed in the transverse plane.

Most simulations have been performed in water in this brachytherapy context, in accordance with the TG-43 formalism. Yet, some recent studies focused on the influence of variations in the elemental composition of human tissues. ${ }^{93,94}$ User interfaces such as BrachyGUI (Ref. 95) and the ALGEBRA (ALgorithm for heterogeneous dosimetry based

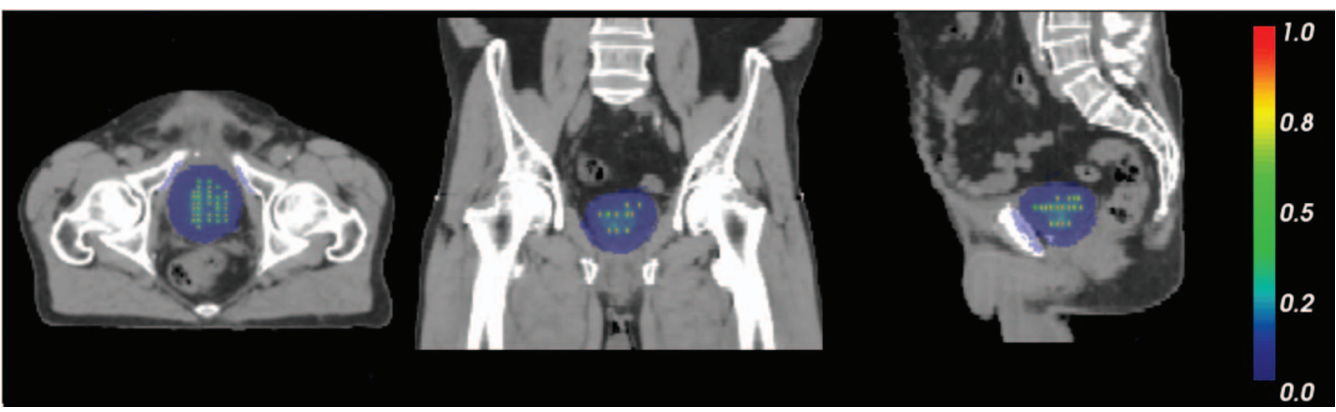

FIG. 2. Dose distribution (normalized to the maximum dose) for a low dose rate brachytherapy treatment using $79{ }^{125}$ I seeds. The Best Medical model 2301

${ }^{125}$ I source (Best Medical International, Springfield, VA.) has been simulated. The seed is made of a double-walled titanium capsule surrounding a tungsten $\mathrm{X}$-ray marker coated with an organic carbon layer impregnated with ${ }^{125} \mathrm{I}$. 
on GEANT4 for BRAchytherapy) platform ${ }^{96}$ have been proposed.

Considering brachytherapy using beta sources, GATE was shown to agree with EGSnrc within less than 3\% in the maximum absorbed dose estimates between $50 \mathrm{keV}$ and $4 \mathrm{MeV}$, while pencil beam kernels agree within less than $4 \%$ between $15 \mathrm{keV}$ and $20 \mathrm{MeV}^{43}$ These results suggest that with an appropriate implementation of the electron multiple scattering algorithms, reliable results are obtained. This is contrary to previously reported disagreements between results obtained with GEANT4 using the low energy package (version 4.1 and later) and with other well-validated MC codes like PENELOPE..$^{97}$ This seemingly contraditory results only emphasize the fact that electron transport parameters should be set very carefully. The PENELOPE package used for GEANT4 simulations regarding electron brachytherapy ${ }^{90}$ is included in the GEANT4 distribution and is thus available for GATE simulations.

\section{C. Intraoperative radiotherapy}

The main objective of IORT is to perform radiotherapy during surgery, directly after the removal of the tumor, to reduce the probability of recurrence. As an example, the INTRABEAM ${ }^{T M}$ (Carl Zeiss Meditec, Oberkochen, Germany) is a widely used IORT device. It is based on the use of a $50 \mathrm{keV}$ x-ray beam delivering nonfractionated doses of 5-20 Gy. Different applicators can be attached to the $\mathrm{x}$-ray source in order to enable isotropic dose delivery depending on the clinical application. For example, for breast cancer treatment a single dose of $20 \mathrm{~Gy}$ is typically delivered to every patient irrespective of individual tissue characteristics or tumor location. A Monte Carlo dose planning system based on GATE has been recently proposed for this device. ${ }^{98}$ GATE allowed for a detailed modeling of the system, including the applicators for both breast cancer and kyphoplastybased IORT. For model validation, the depth-dose curve and anisotropy function were measured in a water phantom specifically designed for measuring the low energy $x$-ray source of the system by means of a soft $x$-ray ionization chamber. These measurements were compared with a GATE simulation of the same water phantom. The depth-dose curve and anisotropy function showed good agreement in water $(<5 \%)$. A breast cancer patient was also scanned by CT with the applicator in place. In between the CT acquisition and the irradiation, a number of thermoluminescent dosimeters (TLDs) were placed on the patient skin at fixed distances around the applicator. The CT scan was subsequently incorporated into the proposed GATE based IORT dosimetry platform and doses at the same location as those of the TLDs were compared to the measured absorbed doses. The simulated and measured doses were found equal to within $1 \%$ (considering $1 \times 10^{9}$ simulated particles), suggesting that GATE can be reliably used for such IORT treatment planning.

\section{D. External beam radiotherapy}

Dosimetric accuracy in advanced techniques of RT such as IMRT, arc-therapy, CyberKnife, TomoTherapy, DMLC, and others is necessary to ensure reliable patient treatment. MC simulations have been extensively used in this field and interested readers may, for example, refer to Ref. 99. Different MC codes have been employed for modeling photon and electron based RT, ${ }^{1,100-106}$ and the use of GATE in that context is rather recent.

Specific tools such as absorbed dose scoring (DoseActor) and Phase-Space (PhS, PhaseSpaceActor) management were implemented within GATE V6 to facilitate the use of GATE for modeling photon and electron based RT. The first study performed with GATE V6 for EBRT demonstrated the feasibility of simulating the whole RT experiment within GATE. ${ }^{107}$ The different physical components of an ELEKTA linear accelerator were simulated and the proposed model was validated through a phantom-based dosimetry study. Three major components were included in the photon fluence model: the target, the primary collimator, and the flattening filter. The model was validated by comparing simulations with various depth-dose and dose profiles measured in water. Simulations and measurements in water agreed well, with relative differences of $1 \%$ and $2 \%$ for depth-dose and dose profiles, respectively. Gamma index comparisons led to more than $90 \%$ of the points for all simulations within the $3 \% / 3 \mathrm{~mm}$ gamma criterion.

In Ref. 108, a $6 \mathrm{MV}$ photon beam linear accelerator (Siemens Oncor Impression) was modeled and validated using percent depth-dose profile in water and tissue-equivalent phantoms. Simulations and measurements were also compared in terms of absolute and relative absorbed doses using IMRT dedicated quality assurance phantoms and patient datasets. In the validation of the accelerator model, tissuephantom ratios obtained from GATE and from measurements were $0.67 \pm 0.063$ and 0.68 , respectively. In terms of percent depth-dose and transverse profiles, discrepancies ranged from $0.04 \%$ to $0.1 \%$ and from $0.07 \%$ to $0.2 \%$, respectively. When comparing absolute absorbed doses between simulated and measured beams based on the IMRT simulations using seven patient datasets, GATE yielded a relative difference of $0.43 \%$ $\pm 0.25 \%$. For the whole set of beams that were studied, the mean gamma-index was $0.5 \pm 0.152$, and $90.8 \% \pm 0.6 \%$ of the measurement points satisfied the 5\% criterion in absorbed dose and the $4 \mathrm{~mm}$ criterion in distance criterion.

\section{E. Particle therapy}

As in conventional external beam therapy, MC simulations in particle therapy are generally used to characterize the treatment beam and compute absorbed dose distributions. To the best of our knowledge, only few publications have used GATE for beam line simulations in hadrontherapy to date.

In Ref. 109, the authors compared GATE (based on GEANT4 V9.2) to experimental measurements for a 100-203 MeV proton beam and reported a $0.3 \mathrm{~mm}$ range and $1 \%$ dose accuracy. However, differences in transverse profiles were up to $15 \%$. Further investigations showed that dose discrepancies of more than $8 \%$ were observed between GEANT4 single and multiple scattering models suggesting 


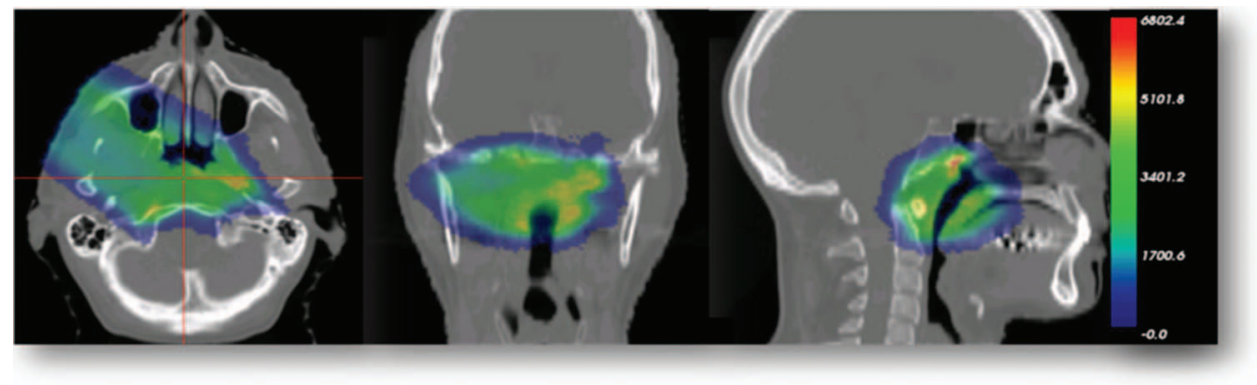

FIG. 3. Absorbed dose distribution (arbitrary unit) of a proton treatment plan computed with GATE. The plan is composed of two fields (single field shown here) with about 30 energy layers per field, with an energy range of 101-135 MeV, 1547 spots in total, and a spot sampling of $8 \mathrm{~mm}$.

that GEANT4 tends to underestimate lateral absorbed dose spreading. The authors also found that the GEANT4 precompound model for inelastic hadronic collisions led to slightly more accurate results than the Binary Cascade model.

In Ref. 29, the authors proposed a methodology to use GATE V6.1 for modeling an IBA scanned proton beam delivery system without beam line description, using only measured data acquired for clinical commissioning. MC simulations were compared to measured data in various configurations. Pristine Bragg peaks were reproduced to within a $0.7 \mathrm{~mm}$ range and $0.2 \mathrm{~mm}$ spot size accuracy, while a $32 \mathrm{~cm}$ range SOBP with $10 \mathrm{~cm}$ modulation was reproduced to within $0.8 \mathrm{~mm}$ range accuracy and a maximum point-to-point dose difference of less than $2 \%$. A 2D test pattern consisting of a combination of homogeneous and high-gradient dose regions passed a $2 \% / 2 \mathrm{~mm}$ gamma index comparison for $97 \%$ of the points. In Ref. 23, this model was used to compute the absorbed dose distribution of a complete treatment plan that was then compared to the one obtained with a commercial TPS (XiO, Elekta). A satisfactory agreement was obtained between $\mathrm{XiO}$ and GATE, with more than $95 \%$ of the points passing a $2 \% / 2 \mathrm{~mm}$ gamma evaluation. Yet, a maximum stopping power difference of $3 \%$ was observed in human tissue of $0.9 \mathrm{~g} \mathrm{~cm}^{3}$ density and led to a range shift of 1-2 mm. Discrepancies near heterogeneous regions (gas in the rectum) were also observed. Figure 3 illustrates the dose distribution resulting from a proton treatment.

Before GATE was used for particle therapy applications, many simulations regarding particle therapy and involving GEANT4 were published, establishing the good adequacy of this tool box in the particle therapy field. For example, Paganetti et al. ${ }^{110,111}$ used GEANT4 V5.2 to model and validate the treatment nozzle of the Northeast Proton Therapy Centre (NPTC), including an explicit model of the temporal variation of the beam energy by the range modulator wheel. ${ }^{112}$ Since 2004, an official hadrontherapy GEANT4 example is also available with the source code, ${ }^{113}$ describing the $62 \mathrm{MeV}$ proton therapy beam line of the CATANA facility (Centro di AdroTerapia e Applicazioni Nucleari Avanzate). GEANT4 is also used in the VMCpro code dedicated to treatment planning in proton beam therapy. ${ }^{114-116}$ GEANT4 was also used to describe magnetic beam scanning for proton ${ }^{117}$ and carbon ${ }^{118,119}$ compared a complete proton treatment plan calculated with GEANT4 V9.3.p01 with that from a TPS. All these contributions provide a solid background to support the idea that GATE can play a significant role in particle therapy applications in the future. Agreements between computed dose and experimental measurements were usually very good, with range accuracy to within a $\mathrm{mm}$. Yet, discrepancies in lateral dose spreading have been reported in several instances and should be investigated carefully.

Recently, a dedicated package called TOPAS (Ref. 14) has been described to simplify GEANT4 simulations of proton therapy beam lines. The TOPAS and GATE platforms have similar features and both rely on GEANT4. GATE is open source, while TOPAS is not. TOPAS can efficiently define proton beam lines, while GATE remains a more generic platform and allows for an easy combined simulation of treatments and imaging.

\section{F. In vivo dose monitoring}

\section{F.1. Context}

In particle therapy, in vivo imaging can be used to verify the accuracy of the absorbed dose deposition inside the patient. To that end, 3D maps or 2D profiles of the location of production of the fragmentation products (prompt gammas, annihilation photons, secondary protons, etc.) are measured using an imaging system during or shortly after the irradiation. Possible errors in dose delivery can then be detected by comparing these measured maps with the expected maps of emitted particles simulated based on the treatment plan. ${ }^{120}$ These errors can be due to uncertainties in the conversion from CT data to hadron interaction data, to mispositioning of the patient, to uncertainties in the beam features, and/or to anatomical modifications (e.g., weight loss) between the treatment plan calculation and the treatment delivery.

Imaging-based methods of treatment delivery monitoring heavily rely on simulated reference activity maps. This monitoring approach therefore strongly depends on the accuracy of the MC codes used to calculate these reference maps. As a result, several recent papers focused on the validation of the MC codes used in this field, including FLUKA, ${ }^{13}$ GATE/GEANT4 (Refs. 17, 18, 121, and 122) and MCNPX. ${ }^{11}$ Here, we mostly discuss applications and results obtained with GATE and possibilities offered by GEANT4. 


\section{F.2. Hadron-PET studies}

Among the imaging-based methods for particle therapy delivery monitoring, PET has already been tested clinically. Using PET, $511 \mathrm{keV}$ photons resulting from the annihilation of positrons produced by $\beta+$ emitters (mainly ${ }^{11} \mathrm{C},{ }^{10} \mathrm{C},{ }^{15} 0$, and ${ }^{13} \mathrm{~N}$ ) are detected and provide indirect information regarding dose deposit within the patient.

In Ref. 123, the authors simulated with GATE a carbon ion beam plan coupled with a complete PET ECAT EXACT HR+ system and demonstrated the need for simulating the full PET system instead of only smoothing the simulated positron emitter maps using a Gaussian function. Differences in the distal position of the signal fall-off of $20 \%$ were observed between the full PET system simulation and a simple convolution model. Robert et al. ${ }^{124}$ used GATE to compare five PET system designs for inbeam treatment monitoring and showed that patient mispositioning of the order of $2 \mathrm{~mm}$ could be detected.

The relevance of GATE for these applications obviously depends on the accuracy of the physics models describing the hadronic interactions producing the $\beta+$ emitters that yield the PET signal. Significant efforts have thus been dedicated to the assessment of the hadronic physics model accuracy. In particular, Seravalli et al. ${ }^{125}$ compared $\beta+$ emitter rates and profiles of depth production in a PMMA target using GATE V6.1 (based on GEANT4 V9.2), FLUKA, and MCNPX for proton irradiations. This study showed that, similar to the FLUKA code, ${ }^{120}$ the GEANT4 internal hadronic models used by GATE led to discrepancies when compared to experimental PET results. An alternative method, consisting in convolving the fluence of the protons with experimental cross-sections, was proposed by ${ }^{120}$ and implemented in GATE. ${ }^{125}$ This latter study showed that when the same crosssection datasets are used, the depth profiles and yields computed by the three MC codes are comparable.

Other investigations of the accuracy of the hadronic physics models in the context of particle therapy monitoring have been reported using GEANT4 only. In 2006, Pshenichnov et al. ${ }^{126}$ compared the depth distributions of positron-emitting nuclei produced in a PMMA target obtained experimentally and by simulation. The total production rates were also compared to the FLUKA and POSGEN MC codes. The Binary Cascade model was used in the GEANT4 simulations. For protons and carbon ions, differences lower than $30 \%$ were observed between experimental and GEANT4 total production yields for the most abundant $\beta+$ emitters, namely, ${ }^{11} \mathrm{C}$ and ${ }^{15} \mathrm{O}$. In Ref. 127 , several cross-section datasets available in the literature for the main reaction channels leading to the $\beta+$ emitters production in protontherapy $\left[{ }^{16} 0(\mathrm{p}, \mathrm{pn}){ }^{15} \mathrm{O}\right.$, $\left.{ }^{12} \mathrm{C}(\mathrm{p}, \mathrm{pn}){ }^{11} \mathrm{C},{ }^{16} 0(\mathrm{p}, 3 \mathrm{p} 3 \mathrm{n}){ }^{11} \mathrm{C}\right]$ were investigated. Depending on the cross-section values used for a tissue-equivalent material, range differences up to $5 \mathrm{~mm}$ were observed for a $30 \mathrm{~min}$ acquisition performed $15 \mathrm{~min}$ after the irradiation (off-line imaging protocol). This study highlighted the need for new experimental campaigns to better characterize cross-section values in the energy ranges relevant to medical applications. In Ref. 3, GEANT4 simulations using optimized cross-section datasets as identified in Ref. 127 were employed to compare inroom and offline PET experiments performed after two patient treatments (adenoid cystic carcinoma). In Ref. 128, the accuracy of different hadronic models available in GEANT4 to predict experimentally measured $\beta+$ emitting isotopes and prompt gamma-production rates has been investigated. Simulations reproduced experimental $\beta+$ emitting isotopes production rates within an accuracy of $24 \%$, and the production rate change as a function of depth agreed well with experimental data. By tuning the tolerance factor of the photon evaporation model available in GEANT4, an excellent agreement between simulated and experimental prompt $\gamma$-ray production rates was also achieved.

For this PET-based particle therapy monitoring application, a confounding factor is introduced by the biological washout through the blood flow of the induced $\beta+$ emitters. The biological washout processes have been incorporated into GATE (WashoutActor) and will be available in GATE V7. In particular, the model proposed by Mizuno et al. has been implemented. ${ }^{129}$ This model accounts for the displacement of the $\beta+$ emitters due to the blood flow, the microcirculation and the trapping of the radioisotopes by stable molecules. Biological studies to further improve the modeling of the washout processes implemented in GATE are currently in progress. The influence of these effects on the measured PET activity distributions is being studied.

Recently, ${ }^{130}$ introduced GEANT4 simulated $\beta+$ emitter distributions as inputs for the EGS4 simulation code. This latter code was used to analyze and optimize the parameters of a partial ring scanner suitable for inbeam PET. No information regarding the physical models used in the GEANT4 simulations was given. The authors concluded that with $600 \mathrm{ps}$ timing resolution, at least an angular acceptance of $4 \pi / 3$ was required to achieve satisfactory estimate of the proton range.

\section{F.3. Prompt-gamma studies}

PG imaging is another approach currently explored to monitor dose deposition in hadrontherapy. $5,6,131$ To our knowledge only one paper using GATE has been published so far in this area, ${ }^{132}$ but several GEANT4 studies have been used and give indications regarding the potential of GATE for such simulations.

In Ref. 132, the authors proposed a machine learning approach based on GATE simulations to create optimized treatment-specific classifiers that detect discrepancies between planned and delivered dose. The proposed method could help to evaluate performance and to optimize the design of PG monitoring devices.

Several GEANT4 studies regarding PG imaging-based hadron therapy monitoring focused on the investigation of the GEANT4 accuracy to model prompt gamma emissions. In Ref. 131, prompt gamma energy spectra acquired using a high-purity germanium detector shielded either with lead (passive shielding) or with a Compton suppression system (active shielding) were compared to simulated spectra for a proton irradiation. Results showed that GEANT4 properly predicted the oxygen, carbon, and calcium emission lines. 
However, a precise modeling of the detector electronic, lacking in the paper, was needed to correctly reproduce the measurements for low energies. In Ref. 133, time-energy spectra corresponding to the irradiation of PMMA and water targets by monoenergetic carbon ions were acquired at the GSI and GANIL facilities with collimated scintillators (BaF2 or NaI) and compared with spectra simulated using GEANT4 V9.1 (Binary Cascade model). The simulations overestimated the prompt-gamma detection yields by a factor of 12 , highlighting the need for an improvement of the GEANT4 de-excitation models. Recent results, obtained with GEANT4 V9.4 (Ref. 134) are more promising, but the prompt gamma yield was still overestimated by a factor of 2 .

Although an extensive validation of GEANT4 is still needed to fully trust results in terms of prompt gamma emission predictions, GEANT4 simulations have also been used to investigate the feasibility of new systems to image promptgamma profiles using Compton camera techniques ${ }^{135-137}$ or mechanical collimators such as knife-edge slits and multiparallel slits. ${ }^{138,139}$ A time-resolved simulation was recently published exploring the potential use of time-of-flight rejection to suppress the background signal created by neutrons in prompt-gamma imaging. ${ }^{140}$ To compare pros and cons of PET and gamma prompt imaging, ${ }^{141}$ used GEANT4 V9.0 to study four patient cases treated for head and neck, prostate, spine, and abdomen cancers by proton therapy. The authors analyzed production yields of particles of interest as well as emission maps corresponding to prompt gammas and positron emitters. The biological washout was modeled. Results suggested that when accounting for washout correction and acquisition time delay, production yields corresponding to promptgammas were 60-80 times higher than rates of annihilation photons. For both PET and prompt-gammas, the correlation between the fall-off of the profiles of the secondary particles and the fall-off of the dose was especially intricate. The authors concluded that checking the accuracy of dose deposit only based on the imaging signal (without referring to simulations) would be extremely challenging, and that the simulation of the secondary particle distribution was needed to conclude at the accuracy of the dose delivery with respect to the treatment plan. These GEANT4 results illustrate the type of study that could be conducted with GATE, with the possibility of precisely and easily modeling highly realistic imaging devices. The importance of precise modeling of the detailed features of the imaging devices for ensuring accurate predictions from the simulations has been demonstrated in Ref. 123.

Simulations are also used to explore the feasibility of original methods for image-based hadrontherapy monitoring. In Ref. 7, imaging of secondary protons performed during carbon ion irradiations was investigated. Two detection techniques were studied: the double-proton detection using two forward-located trackers and the single-proton detection in coincidence with the incoming carbon ion detected by means of a beam hodoscope.

Last, some studies only focus on the validation of the GEANT4 hadronic models in the context of image-based therapy monitoring, without considering a specific imaging tech- nique. In Ref. 142, the results obtained with the nuclear models implemented in GATE/GEANT4 and FLUKA were compared by analyzing the angular and energy distributions of secondary particles exiting a homogeneous target of PMMA for a proton and a $\mathrm{C} 12$ beam. Despite the very simple target and set-up, substantial discrepancies were observed between the two codes, especially in terms of produced high energy $(>1 \mathrm{MeV})$ prompt gammas whatever the beam and in term of exiting neutrons for the proton beam. All these results call for further investigation of the physics models and of the impact these models have on the simulated data. Along the same line, but at the GEANT4 level only, ${ }^{143}$ analyzed integral and differential yields of secondary fragments produced during carbon ion irradiations. The yields were simulated using GEANT4 (Binary Cascade and Quantum Molecular Dynamics models) and FLUKA MC codes. A reasonable accuracy was obtained for integrated yields but results suggested that hadronic models of GEANT4 should be improved to correctly reproduce secondary fragment angular distributions. The use of the QMD model to handle ion-ion interactions was also recommended.

A meta-analysis of the results related to the use of GEANT4 for hadrontherapy applications led to the definition of optimized GEANT4 physics lists and parameter settings relevant for imaging-based dose verification methods in hadrontherapy. The parameter settings can be found on the GATE website (http://www.opengatecollaboration.org/ UsersGuide).

\section{DISCUSSION AND CONCLUSION}

To further illustrate our review, we provided access to ready-to-use examples of realistic GATE simulations that include particle tracking in voxelized phantoms based on CT images and aim to provide 3D dose distributions. Five examples corresponding to EBRT (both photon and electron beams), brachytherapy, protontherapy, and molecular RT (MRT) are detailed on the web page of the OpenGate collaboration $^{19}$ (http://wiki.opengatecollaboration.org/ DosiGate). Such examples might guide potential users in the settings of a specific simulation and are used by GATE developers to monitor changes in results introduced by changes in GEANT4 and in GATE versions. All examples were run on the same computer (single core Intel Xeon CPU E5-1660 $3.3 \mathrm{GHz}$ ), and Table I summarizes the number of tracks generated by primary particles (events), the total number of steps (all particles included), the ratio between the numbers of geometrical steps (limited by a geometrical boundary) and physical steps (limited by a physical process), the approximate number of primary events needed to reach $2 \%$ statistical uncertainty in the target, the number of particle per seconds and the total simulation time. Results show that the computing time strongly depends on the physics involved in the simulation. For example, the protontherapy case produced 50 times more particles than the brachytherapy case. Also the ratio between the numbers of geometrical steps and physical steps depends on the energy and type of particles. To the best of our knowledge, this is the first time such a comparative 
TABLE I. Simulation features and time durations for four examples of GATE simulations available online. Columns are: "tracks by p." is the mean number of tracks by primary particle, "Steps by p." is the mean number of steps by primary particle, "Steps phy/geo" is the ratio between the geometrical and physical steps, "uncert 2\%" is the (approximate) number of primary particles needed to reach $2 \%$ statistical uncertainty in the target region, "PPS" is the number of particles per seconds, and "Time" the total needed time to reach $2 \%$ statistical uncertainty.

\begin{tabular}{lccccrc}
\hline \hline Example & $\begin{array}{c}\text { Tracks } \\
\text { by } \mathrm{p} .\end{array}$ & $\begin{array}{c}\text { Steps } \\
\text { by } \mathrm{p} .\end{array}$ & $\begin{array}{c}\text { Steps } \\
\text { phy/geo }\end{array}$ & $\begin{array}{c}\text { Uncert. } \\
2 \%\end{array}$ & PPS & Time \\
\hline EBRT photon & 2.6 & 198 & 0.5 & $6 \mathrm{e} 7$ & 6300 & $2.7 \mathrm{~h}$ \\
EBRT electron & 28.6 & 429 & 16 & $2 \mathrm{e} 8$ & 500 & $4.6 \mathrm{j}$ \\
Brachytherapy & 1.0 & 25 & 0.07 & $5 \mathrm{e} 7$ & 20000 & $45 \mathrm{~min}$ \\
Protontherapy & 58 & 535 & 8.23 & $3 \mathrm{e} 6$ & 270 & $3.1 \mathrm{~h}$ \\
Molecular RT & 1.8 & 65 & 0.55 & $2 \mathrm{e} 8$ & 3500 & $16 \mathrm{~h}$ \\
\hline \hline
\end{tabular}

analysis between different applications, using the same code and the same computer, is presented. It illustrates the computation time to be expected for a given application.

To obtain absorbed dose uncertainties below few percent in MRT dosimetry it is typically necessary to simulate about ten times the primary particles needed to obtain similar uncertainties in EBRT. The reason is mainly related to the different geometries of the radiation sources: while in EBRT the radiation source is well collimated, and the absorbed dose calculation is restricted to a relatively small region, in MRT the source distribution is usually heterogeneous and spread throughout the patient body. The dosimetry of organs with (source) and without (target) specific uptake is of equal importance. In target organs the absorbed dose is mainly due to photons generated in source organs. Since for ballistic reasons only a small percentage of the emitted photon radiation reaches the target organs, the simulation statistics in these regions converge slowly.

The ability of GATE to easily design both imaging and dosimetry simulations in the same framework is of paramount importance in molecular dosimetry as activity quantification is a prerequisite to absorbed dose calculation. It is now possible to consider the simulation of a complete clinical dosimetry study (i.e., from image generation to absorbed dose maps calculation) with the same MC code. This is the aim of a currently ongoing project, DosiTest, ${ }^{69}$ for which the combined modeling of imaging and dosimetry is essential. Similarly, the fact that GATE supports both imaging and RT modeling makes it especially suitable for investigating the emerging field of in vivo dose delivery monitoring in hadrontherapy. Here, GATE may play an important role in the development of dedicated devices for imaging the secondary radiations induced by therapeutic hadrons within the patient. Moreover, the correlation of the dose distribution and the secondary emission reference map on a per-patient basis within a single, user-friendly software framework might prove to be crucial in the clinical evaluation and application of these new RT monitoring approaches.

Although GATE is mostly known for its imaging applications so far, this review aimed at demonstrating its versatility and potentials for dosimetry and radiotherapy applications, based on an already significant amount of results and validation studies. Being an open-source, user-friendly and integrated tool enabling simulations of RT, dosimetry and imaging in the very same framework, it is expected that GATE will play an increasing role in the emerging domain of combined imaging and therapy. Advanced users are welcome to contribute to the source code.

To best meet the needs prompted by these new applications, developments by the OpenGATE collaboration are ongoing. A release supporting CPU and GPU architectures for specific applications (PET and photon RT) is being prepared. ${ }^{35}$ Work is also in progress to include forced detection in GATE for $\mathrm{kV}$ imaging. Speeding up GATE simulations is also considered by combining MC and analytic algorithms within GATE ${ }^{34}$ first for absorbed dose distribution of low energy beams (imaging, synchrotron treatments, small animal treatments).

\section{ACKNOWLEDGMENTS}

This work was supported in part by the European collaboration Envision (Grant Agreement No. 241851), the IMADRON project from INCa, the Labex PRIMES (ANR11-LABX-0063) of Université de Lyon within the program "Investissements d'Avenir" (ANR-11-IDEX-0007) operated by the French National Research Agency (ANR), the Lyric grant INCa-DGOS-4664, the DOSEVAL grant (INCa).

a) Author to whom correspondence should be addressed. Electronic mail: david.sarrut@creatis.insa-lyon.fr

${ }^{1}$ N. Reynaert, S. Vandermarck, D. Schaart, W. Vanderzee, C. Vanvlietvroegindeweij, M. Tomsej, J. Jansen, B. Heijmen, M. Coghe, and C. Dewagter, "Monte carlo treatment planning for photon and electron beams," Radiat. Phys. Chem. 76, 643-686 (2007).

${ }^{2}$ M. J. Murphy, J. M. Balter, S. Balter, J. A. BenComo, I. J. Das, S. B. Jiang, C.-M. Ma, G. H. Olivera, R. F. Rodebaugh, K. J. Ruchala, H. Shirato, and F.-F. Yin, "The management of imaging dose during image-guided radiotherapy: Report of the AAPM Task Group 75," Med. Phys. 34, 40414063 (2007)

${ }^{3}$ X. Zhu, S. España, J. Daartz, N. Liebsch, J. Ouyang, H. Paganetti, T. R. Bortfeld, and G. El Fakhri, "Monitoring proton radiation therapy with in-room PET imaging," Phys. Med. Biol. 56, 4041-4057 (2011).

${ }^{4}$ K. Parodi, T. Bortfeld, and T. Haberer, "Comparison between in-beam and offline positron emission tomography imaging of proton and carbon ion therapeutic irradiation at synchrotron- and cyclotron-based facilities," Int. J. Radiat. Oncol., Biol., Phys. 71, 945-956 (2008).

${ }^{5}$ C.-H. Min, C. H. Kim, M.-Y. Youn, and J.-W. Kim, "Prompt gamma measurements for locating the dose falloff region in the proton therapy," Appl. Phys. Lett. 89, 183517 (2006).

${ }^{6}$ E. Testa, M. Bajard, M. Chevallier, D. Dauvergne, F. Le Foulher, N. Freud, J. Létang, J. Poizat, C. Ray, and M. Testa, "Monitoring the bragg peak location of $73 \mathrm{MeV} / \mathrm{u}$ carbon ions by means of prompt gamma-ray measurements," Appl. Phys. Lett. 93, 093506 (2008).

${ }^{7}$ P. Henriquet, E. Testa, M. Chevallier, D. Dauvergne, G. Dedes, N. Freud, J. Krimmer, J. M. Létang, C. Ray, M.-H. Richard, and F. Sauli, "Interaction vertex imaging (IVI) for carbon ion therapy monitoring: A feasibility study," Phys. Med. Biol. 57, 4655-4669 (2012).

${ }^{8}$ M. Ljungberg and S. E. Strand, "A Monte Carlo program for the simulation of scintillation camera characteristics," Comput Methods Programs Biomed 29, 257-272 (1989).

${ }^{9}$ R. Harrison and S. Vannoy, "Preliminary experience with the photon history generator module of a public-domain simulation system for emission tomography," in Proceedings of the IEEE Nuclear Science Symposium and Medical Imaging Conference (1993), pp. 1154-1158. 
${ }^{10}$ M. Cañadas, P. Arce, and P. Rato Mendes, "Validation of a small-animal pet simulation using gamos: A GEANT4-based framework," Phys. Med. Biol. 56, 273-288 (2011).

${ }^{11}$ LANL, MCNPX 2.6.0 Users's Guide, Technical Report No. LA-CP-07 $1473,2008$.

${ }^{12}$ G. Battistoni, S. Muraro, P. Sala, F. Cerutti, A. Ferrari, S. Roesler, A. Fasso, and J. Ranft, "The FLUKA code: Description and benchmarking," in Hadronic Shower Simulations Workshop, edited by M. Albrow and R. Raja (AIP Conference, Fermilab, Batavia, IL, 2006), Vol. 896, pp. 31-49.

${ }^{13}$ A. Ferrari, P. Sala, A. Fasso, and J. Ranft, FLUKA: A Multi-Particle Transport Code, Technical Report No. INFN/TC 05/11, SLAC-R-773, CERN2005-10, 2005.

${ }^{14}$ J. Perl, J. Shin, B. Faddegon, and H. Paganetti, "TOPAS: An innovative proton Monte Carlo platform for research," Med. Phys. 39, 6818-6837 (2012).

${ }^{15}$ B. R. B. Walters, I. Kawrakow, and D. W. O. Rogers, "History by history statistical estimators in the beam code system," Med. Phys. 29, 2745-2752 (2002).

${ }^{16}$ I. Kawrakow and M. Fippel, "Investigation of variance reduction techniques for Monte Carlo photon dose calculation using XVMC," Phys. Med. Biol. 45, 2163-2183 (2000).

${ }^{17}$ S. Jan, G. Santin, D. Strul, S. Staelens, K. Assié, D. Autret, S. Avner, R. Barbier, M. Bardiès, P. M. Bloomfield, D. Brasse, V. Breton, P. Bruyndonckx, I. Buvat, A. F. Chatziioannou, Y. Choi, Y. H. Chung, C. Comtat, D. Donnarieix, L. Ferrer, S. J. Glick, C. J. Groiselle, D. Guez, P.-F. F. Honore, S. Kerhoas-Cavata, A. S. Kirov, V. Kohli, M. Koole, M. Krieguer, D. J. V. D. Laan, F. Lamare, G. Largeron, C. Lartizien, D. Lazaro, M. C. Maas, L. Maigne, F. Mayet, F. Melot, C. Merheb, E. Pennacchio, J. Perez, U. Pietrzyk, F. R. Rannou, M. Rey, D. R. Schaart, C. R. Schmidtlein, L. Simon, T. Y. Song, J.-M. M. Vieira, D. Visvikis, R. V. D. Walle, E. Wieërs, C. Morel, K. Assie, M. Bardies, D. J. van der Laan, R. Van de Walle, and E. Wieers, "Gate: A simulation toolkit for PET and SPECT," Phys. Med. Biol. 49, 4543-4561 (2004).

${ }^{18}$ S. Jan, D. Benoit, E. Becheva, T. Carlier, F. Cassol, P. Descourt, T. Frisson, L. Grevillot, L. Guigues, L. Maigne, C. Morel, Y. Perrot, N. Rehfeld, D. Sarrut, D. R. Schaart, S. Stute, U. Pietrzyk, D. Visvikis, N. Zahra, and I. Buvat, "GATE V6: A major enhancement of the gate simulation platform enabling modelling of CT and radiotherapy," Phys. Med. Biol. 56, 881-901 (2011).

${ }^{19}$ The OpenGate Collaboration, http://www.opengatecollaboration.org, 2014.

${ }^{20}$ The OpenGate Collaboration, http://git.opengatecollaboration.org/git/ opengate-public.git, 2014.

${ }^{21}$ I. J. Chetty, M. Rosu, M. L. Kessler, B. A. Fraass, R. K. Ten Haken, F.-M. S. Kong, and D. L. McShan, "Reporting and analyzing statistical uncertainties in Monte Carlo-based treatment planning," Int. J. Radiat. Oncol., Biol., Phys. 65, 1249-1259 (2006).

${ }^{22} \mathrm{H}$. Paganetti, "Dose to water versus dose to medium in proton beam therapy," Phys. Med. Biol. 54, 4399-4421 (2009).

${ }^{23}$ L. Grevillot, D. Bertrand, F. Dessy, N. Freud, and D. Sarrut, "Gate as a GEANT4-based Monte Carlo platform for the evaluation of proton pencil beam scanning treatment plans," Phys. Med. Biol. 57, 4223-4244 (2012).

${ }^{24} \AA$. C. Tedgren and G. A. Carlsson, "Specification of absorbed dose to water using model-based dose calculation algorithms for treatment planning in brachytherapy," Phys. Med. Biol. 58, 2561-2579 (2013).

${ }^{25} \mathrm{H}$. Jiang and H. Paganetti, "Adaptation of GEANT4 to Monte Carlo dose calculations based on CT data," Med. Phys. 31, 2811-2818 (2004).

${ }^{26}$ H. Jiang, J. Seco, and H. Paganetti, "Effects of Hounsfield number conversion on CT based proton Monte Carlo dose calculations," Med. Phys. 34, 1439-1449 (2007)

${ }^{27}$ W. Schneider, T. Bortfeld, and W. Schlegel, "Correlation between CT numbers and tissue parameters needed for Monte Carlo simulations of clinical dose distributions," Phys. Med. Biol. 45, 459-478 (2000).

${ }^{28}$ D. Sarrut and L. Guigues, "Region-oriented CT image representation for reducing computing time of Monte Carlo simulations," Med. Phys. 35, 1452-1463 (2008)

${ }^{29}$ L. Grevillot, T. Frisson, D. Maneval, N. Zahra, J. Badel, and D. Sarrut, "Simulation of a $6 \mathrm{MV}$ Elekta Precise linac photon beam using GATE/GEANT4,” Phys. Med. Biol. 56, 903-918 (2011).

${ }^{30}$ N. S. Rehfeld, S. Stute, J. Apostolakis, M. Soret, and I. Buvat, "Introducing improved voxel navigation and fictitious interaction tracking in gate for enhanced efficiency," Phys. Med. Biol. 54, 2163-2178 (2009).
${ }^{31}$ J. J. DeMarco, I. J. Chetty, and T. D. Solberg, "A Monte Carlo tutorial and the application for radiotherapy treatment planning," Med. Dosim. 27, 4350 (2002).

${ }^{32}$ A. Mittone et al., "An efficient numerical tool for dose deposition prediction applied to synchrotron medical imaging and radiation therapy," J. Synchrotron Radiat. 20, 785-792 (2013).

${ }^{33}$ G. Poludniowski, P. M. Evans, V. N. Hansen, and S. Webb, "An efficient Monte Carlo-based algorithm for scatter correction in keV cone-beam CT," Phys. Med. Biol. 54, 3847-3864 (2009).

${ }^{34}$ F. Smekens, N. Freud, J. M. Létang, J.-F. Adam, C. Ferrero, H. Elleaume, A. Bravin, F. Estève, and D. Babot, "Simulation of dose deposition in stereotactic synchrotron radiation therapy: A fast approach combining Monte Carlo and deterministic algorithms," Phys. Med. Biol. 54, 46714685 (2009)

${ }^{35}$ J. Bert, H. Perez-Ponce, Z. E. Bitar, S. Jan, Y. Boursier, D. Vintache, A. Bonissent, C. Morel, D. Brasse, and D. Visvikis, "GEANT4-based Monte Carlo simulations on GPU for medical applications," Phys. Med. Biol. 58, 5593-5611 (2013).

${ }^{36}$ M. J. Berger, Improved Point Kernels for Electron and Beta-Ray Dosimetry (US Department of Commerce, National Bureau of Standards NBSIR, Washington DC, 1973), pp. 73-107.

${ }^{37}$ D. J. Simpkin and T. R. Mackie, "EGS4 Monte Carlo determination of the beta dose kernel in water," Med. Phys. 17, 179-186 (1990).

${ }^{38}$ M. Bardiès, C. S. Kwok, and G. Sgouros, "Dose point-kernel for radionuclide dosimetry," in Therapeutic Applications of Monte Carlo Calculations in Nuclear Medicine, edited by H. Zaidi and G. Sgouros (Institute of Physics Publishing, London, 2002), Chap. 7.

${ }^{39}$ H. Uusijarvi, N. Chouin, P. Bernhardt, L. Ferrer, M. Bardies, and E. Forssell-Aronsson, "Comparison of electron dose-point kernels in water generated by the Monte Carlo codes, PENELOPE, GEANT4, MCNPX, and ETRAN," Cancer Biother. Radiopharm. 24, 461-467 (2009).

${ }^{40}$ F. Botta, A. Mairani, G. Battistoni, M. Cremonesi, A. Di Dia, A. Fasso, A. Ferrari, M. Ferrari, G. Paganelli, G. Pedroli, and M. Valente, "Calculation of electron and isotopes dose point kernels with FLUKA Monte Carlo code for dosimetry in nuclear medicine therapy," Med. Phys. 38, 3944-3954 (2011).

${ }^{41}$ J. Seco and F. Verhaegen, Monte Carlo Techniques in Radiation Therapy (CRC Press, 2013), Chap. 15, p. 223.

${ }^{42}$ L. Ferrer, N. Chouin, A. Bitar, A. Lisbona, and M. Bardies, "Implementing dosimetry in GATE: dose-point kernel validation with GEANT4 4.8.1," Cancer Biother. Radiopharm. 22, 125-129 (2007)

${ }^{43}$ L. Maigne, Y. Perrot, D. R. Schaart, D. Donnarieix, and V. Breton, "Comparison of GATE/GEANT4 with EGSnrc and MCNP for electron dose calculations at energies between $15 \mathrm{keV}$ and $20 \mathrm{MeV}$," Phys. Med. Biol. 56, 811-827 (2011)

${ }^{44}$ D. R. Schaart, J. T. M. Jansen, J. Zoetelief, and P. F. A. de Leege, "A comparison of MCNP4C electron transport with ITS 3.0 and experiment at incident energies between $100 \mathrm{keV}$ and $20 \mathrm{MeV}$ : Influence of voxel size, substeps and energy indexing algorithm," Phys. Med. Biol. 47, 1459-1484 (2002).

${ }^{45}$ G. Loudos, I. Tsougos, S. Boukis, N. Karakatsanis, P. Georgoulias, K. Theodorou, K. Nikita, and C. Kappas, "A radionuclide dosimetry toolkit based on material-specific Monte Carlo dose kernels," Nucl. Med. Commun. 30, 504-512 (2009).

${ }^{46}$ P. Papadimitroulas, G. Loudos, G. Nikiforidis, and G. Kagadis, "A dose point kernel database using GATE Monte Carlo simulation toolkit for nuclear medicine applications: Comparison with other Monte Carlo codes," Med. Phys. 39, 5238-5247 (2012)

${ }^{47}$ E. Amato, A. Italiano, F. Minutoli, and S. Baldari, "Use of the GEANT4 Monte Carlo to determine three-dimensional dose factors for radionuclide dosimetry," Nucl. Instrum. Methods Phys. Res., Sect. A 708, 15-18 (2013)

${ }^{48}$ N. Chouin, K. Bernardeau, F. Davodeau, M. Cherel, A. FaivreChauvet, M. Bourgeois, C. Apostolidis, A. Morgenstern, A. Lisbona, and M. Bardies, "Evidence of extranuclear cell sensitivity to alpha-particle radiation using a microdosimetric model. I. Presentation and validation of a microdosimetric model," Radiat. Res. 171, 657-663 (2009).

${ }^{49}$ L. Thijssen, D. R. Schaart, D. de Vries, A. Morgenstern, F. Bruchertseifer, and A. G. Denkova, "Polymersomes as nano-carriers to retain harmful recoil nuclides in alpha radionuclide therapy: A feasibility study," Radiochim. Acta 100, 473-482 (2012).

${ }^{50}$ S. Incerti, A. Ivanchenko, M. Karamitros, A. Mantero, P. Moretto, H. N. Tran, B. Mascialino, C. Champion, V. N. Ivanchenko, M. A. Bernal, 
Z. Francis, C. Villagrasa, G. Baldacchino, P. Gueye, R. Capra, P. Nieminen, and C. Zacharatou, "Comparison of GEANT4 very low energy cross section models with experimental data in water," Med. Phys. 37, 46924708 (2010).

${ }^{51}$ C. Villagrasa, Z. Francis, and S. Incerti, "Physical models implemented in the GEANT4-DNA extension of the GEANT4 toolkit for calculating initial radiation damage at the molecular level," Radiat. Protect. Dosim. 143, 214-218 (2011)

${ }^{52}$ R. Loevinger, T. Budinger, and E. Watson, MIRD Primer for Absorbed Dose Calculations. Revised, Technical Report (The Society of Nuclear Medicine, New York, 1991)

${ }^{53}$ M. Stabin, "Nuclear medicine dosimetry," Phys. Med. Biol. 51, R187R202 (2006)

${ }^{54}$ M. G. Stabin, M. A. Emmons, W. P. Segars, and M. J. Fernald, "Realistic reference adult and paediatric phantom series for internal and external dosimetry," Radiat. Protect. Dosim. 149, 56-59 (2012).

${ }^{55}$ M. A. Keenan, M. G. Stabin, W. P. Segars, and M. J. Fernald, "RADAR realistic animal model series for dose assessment," J. Nucl. Med. 51, 471476 (2010)

${ }^{56}$ R. Taschereau and A. F. Chatziioannou, "Monte Carlo simulations of absorbed dose in a mouse phantom from 18-fluorine compounds," Med. Phys. 34, 1026-1036 (2007).

${ }^{57}$ W. Segars, B. Tsui, and E. Frey, "Development of a 4D digital mouse phantom for molecular imaging research," Mol. Imaging Biol. 6, 149-159 (2004).

${ }^{58}$ T. Mauxion, J. Barbet, J. Suhard, J.-P. Pouget, M. Poirot, and M. Bardiès, "Improved realism of hybrid mouse models may not be sufficient to generate reference dosimetric data," Med. Phys. 40, 052501 (11pp.) (2013).

${ }^{59}$ T. Mauxion, J. Barbet, J. Suhard, and M. Bardiès, "Validation of a scaled digital phantom Moby based on 18F S-values calculation with Monte Carlo codes GATE v6.1 and MCNPX v2.7," in Proceedings of the European Association of Nuclear Medicine Congress, 2012.

${ }^{60}$ T. Mauxion, J. Barbet, J. Suhard, and M. Bardiès, "Reference rodent Svalues based on Monte Carlo simulation with GATE (v6.1) for radionuclides of interest in $\mu$-PET imaging," in Proceedings of the European Association of Nuclear Medicine Congress, 2012.

${ }^{61} \mathrm{C}$. Williams, D. Burckhardt, and J. Engdahl, "Evaluation and validation of GATE-based absorbed dose calculation for 3D patient-specific internal dosimetry," Med. Phys. 33, 2098 (2006).

${ }^{62}$ A. A. Parach, H. Rajabi, and M. A. Askari, "Assessment of MIRD data for internal dosimetry using the GATE Monte Carlo code," Radiat. Environ. Biophys. 50, 441-450 (2011).

${ }^{63}$ W. S. Snyder, H. L. Fisher, M. R. Ford, and G. G. Warner, "Estimates of absorbed fractions for monoenergetic photon sources uniformly distributed in various organs of a heterogeneous phantom," J. Nucl. Med. 3, 7-52 (1969).

${ }^{64}$ S. Chiavassa, M. Bardies, F. Guiraud-Vitaux, D. Bruel, J. R. Jourdain, D. Franck, and I. Aubineau-Laniece, "Oedipe: A personalized dosimetric tool associating voxel-based models with mcnpx," Cancer Biother. Radiopharm. 20, 325-332 (2005).

${ }^{65}$ M. G. Stabin and H. Yoriyaz, "Photon specific absorbed fractions calculated in the trunk of an adult male voxel-based phantom," Health Phys. 82, 21-44 (2002).

${ }^{66}$ H. Yoriyaz, A. dos Santos, M. G. Stabin, and R. Cabezas, "Absorbed fractions in a voxel-based phantom calculated with the MCNP-4B code," Med. Phys. 27, 1555-1562 (2000).

${ }^{67}$ I. Zubal, C. Harrell, and E. Smith, "Computerized three-dimensional segmented human anatomy," Med. Phys. 21, 298-305 (1994).

${ }^{68}$ E. Saeedzadeh, S. Sarkar, A. Abbaspour Tehrani-Fard, M. R. Ay, H. R. Khosravi, and G. Loudos, "3D calculation of absorbed dose for 131Itargeted radiotherapy: A Monte Carlo study," Radiat. Protect. Dosim. 150, 298-305 (2012).

${ }^{69}$ D. Villoing, B. McParland, J. Suhard, L. Ferrer, and M. Bardiès, "Dosimetric calculation with Monte Carlo simulation of a PET radiotracer: Comparison between the standard and the personalized approach," in Proceedings of the European Association of Nuclear Medicine Congress, 2012.

${ }^{70} \mathrm{~J}$. Seco and F. Verhaegen, "Monte Carlo methods and applications for brachytherapy dosimetry and treatment planning," Monte Carlo Techniques in Radiation Therapy (CRC Press, 2013), Chap. 9, p. 125.

${ }^{71}$ M. J. Rivard, B. M. Coursey, L. A. DeWerd, W. F. Hanson, M. Saiful Huq, G. S. Ibbott, M. G. Mitch, R. Nath, and J. F. Williamson, "Update of AAPM Task Group No. 43 Report: A revised AAPM protocol for brachytherapy dose calculations," Med. Phys. 31, 633-674 (2004).
${ }^{72}$ L. Beaulieu, A. Carlsson Tedgren, J.-F. Carrier, S. D. Davis, F. Mourtada, M. J. Rivard, R. M. Thomson, F. Verhaegen, T. A. Wareing, and J. F. Williamson, "Report of the task group 186 on model-based dose calculation methods in brachytherapy beyond the tg- 43 formalism: Current status and recommendations for clinical implementation," Med. Phys. 39, 6208-6236 (2012)

${ }^{73}$ J.-F. Carrier and L. Beaulieu, "Impact of interseed attenuation and tissue composition for permanent," Med. Phys. 33, 595-604 (2006).

${ }^{74}$ H. Afsharpour, M. D’Amours, B. Coté, J.-F. Carrier, F. Verhaegen, and L. Beaulieu, "A Monte Carlo study on the effect of seed design on the interseed attenuation in permanent prostate implants," Med. Phys. 35, 36713681 (2008)

${ }^{75}$ R. Nath, H. Amols, C. Coffey, D. Duggan, S. Jani, Z. Li, M. Schell, C. Soares, J. Whiting, P. E. Cole, I. Crocker, and R. Schwartz, "Intravascular brachytherapy physics: Report of the AAPM Radiation Therapy Committee Task Group No. 60,” Med. Phys. 26, 119-152 (1999).

${ }^{76}$ S.-T. Chiu-Tsao, D. R. Schaart, C. G. Soares, and R. Nath, "Dose calculation formalisms and consensus dosimetry parameters for intravascular brachytherapy dosimetry: Recommendations of the AAPM Therapy Physics Committee Task Group No. 149," Med. Phys. 34, 4126-4157 (2007)

${ }^{77}$ C. O. Thiam, V. Breton, D. Donnarieix, B. Habib, and L. Maigne, "Validation of a dose deposited by low-energy photons using GATE/GEANT4," Phys. Med. Biol. 53, 3039-3055 (2008).

${ }^{78}$ D. Granero, J. Pérez-Calatayud, F. Ballester, E. Casal, and J. M. De Frutos, "Dosimetric study of the $15 \mathrm{~mm}$ ROPES eye plaque," Med. Phys. 31, 3330-3336 (2004).

${ }^{79}$ J. Pérez-Calatayud, D. Granero, and F. Ballester, "Phantom size in brachytherapy source dosimetric studies," Med. Phys. 31, 2075-2081 (2004).

${ }^{80}$ J. Pérez-Calatayud, D. Granero, E. Casal, F. Ballester, and V. Puchades, "Monte Carlo and experimental derivation of TG43 dosimetric parameters for CSM-type Cs-137 sources,” Med. Phys. 32, 28-36 (2005).

${ }^{81}$ A. S. Meigooni, C. Wright, R. a. Koona, S. B. Awan, D. Granero, J. PerezCalatayud, and F. Ballester, "TG-43 U1 based dosimetric characterization of model 67-6520 Cs-137 brachytherapy source," Med. Phys. 36, 47114712 (2009).

${ }^{82}$ S. S. O. Fonseca-Rodrigues, M. C. Martins, M. Begalli, P. P. Q. Filho, and D. D. Souza-Santos, "Calculation of dosimetry parameters for 192Ir and 125I brachytherapy sources using GEANT4," in Proceeding of the Nuclear Science Symposium Conference Record, 2010.

${ }^{83}$ S. S. O. Fonseca-Rodrigues, M. Begalli, P. P. Q. Filho, and D. SouzaSantos, "Monte Carlo simulation of an Ir-192 brachytherapy source spectra, geometry and anysotropy factors using GEANT4 code," in Proceeding of the Nuclear Science Symposium Conference Record, 2008.

${ }^{84}$ D. Granero, J. Pérez-Calatayud, and F. Ballester, "Monte Carlo calculation of the TG-43 dosimetric parameters of a new BEBIG Ir-192 HDR source," Radiother. Oncol. 76, 79-85 (2005).

${ }^{85}$ D. Granero, J. Vijande, F. Ballester, and M. J. Rivard, "Dosimetry revisited for the hdr 192Ir brachytherapy source model mHDR-v2," Med. Phys. 38, 487-494 (2011).

${ }^{86}$ D. Granero, J. Pérez-Calatayud, E. Casal, F. Ballester, and J. Venselaar, "A dosimetric study on the Ir-192 high dose rate flexisource," Med. Phys. 33, 4578-4582 (2006)

${ }^{87}$ D. Granero, J. Pérez-Calatayud, J. Gimeno, F. Ballester, E. Casal, V. Crispín, and R. Van Der Laarse, "Design and evaluation of a hdr skin applicator with flattening filter," Med. Phys. 35, 495-503 (2008).

${ }^{88}$ J. Pérez-Calatayud, D. Granero, F. Ballester, V. Crispín, and R. Van Der Laarse, "Technique for routine output verification of leipzig applicators with a well chamber," Med. Phys. 33, 16-20 (2006).

${ }^{89}$ J. Pérez-Calatayud, D. Granero, F. Ballester, V. Puchades, E. Casal, A. Soriano, and V. Crispín, “A dosimetric study of Leipzig applicators," Int. J. Radiat. Oncol., Biol., Phys. 62, 579-584 (2005).

${ }^{90}$ S. Enger, M. D'Amours, and L. Beaulieu, "Modeling a hypothetical 170 Tm source for brachytherapy applications," Med. Phys. 38, 5307$5310(2011)$

${ }^{91}$ S. Enger, H. Lundqvist, M. D'Amours, and L. Beaulieu, "Exploring (57)Co as a new isotope for brachytherapy applications," Med. Phys. 39, 2342-2345 (2012).

${ }^{92}$ D. Liu, E. Poon, M. Bazalova, B. Reniers, M. Evans, T. Rusch, and F. Verhaegen, "Spectroscopic characterization of a novel electronic brachytherapy system," Phys. Med. Biol. 53, 61-75 (2008). 
${ }^{93}$ G. Landry, B. Reniers, L. Murrer, L. Lutgens, E. Bloemen-Van Gurp, J.P. Pignol, B. Keller, L. Beaulieu, and F. Verhaegen, "Sensitivity of low energy brachytherapy Monte Carlo dose calculations to uncertainties in human tissue composition," Med. Phys. 37, 5188-5198 (2010).

${ }^{94}$ G. Landry, P. V. Granton, B. Reniers, M. C. Öllers, L. Beaulieu, J. E. Wildberger, and F. Verhaegen, "Simulation study on potential accuracy gains from dual energy CT tissue segmentation for low-energy brachytherapy Monte Carlo dose calculations," Phys. Med. Biol. 56, 6257 (2011).

${ }^{95}$ E. Poon, Y. Le, J. F. Williamson, and F. Verhaegen, "BrachyGUI: An adjunct to an accelerated Monte Carlo photon transport code for patientspecific brachytherapy dose calculations and analysis," J. Phys.: Conf. Ser. 102, 012018 (2008).

${ }^{96}$ H. Afsharpour, G. Landry, M. D'Amours, S. Enger, B. Reniers, E. Poon, J.-F. Carrier, F. Verhaegen, and L. Beaulieu, "Algebra: Algorithm for the heterogeneous dosimetry based on GEANT4 for brachytherapy," Phys. Med. Biol. 57, 3273-3280 (2012).

${ }^{97}$ J. Torres, M. J. Buades, J. F. Almansa, R. Guerrero, and A. M. Lallena, "Dosimetry characterization of 32P intravascular brachytherapy source wires using Monte Carlo codes PENELOPE and GEANT4," Med. Phys. 31, 296-304 (2004).

${ }^{98}$ D. Bouzid, N. Boussion, S. Benhalouche, O. Pradier, A.-S. Lucia, and D. Visvikis, "[simulation Monte Carlo sur GATE V6.1 d'un appareil peropératoire intrabeam pour le cancer du sein]," Cancer Radiothér. 16, 528529 (2012).

${ }^{99}$ J. Seco and F. Verhaegen, "Monte Carlo modeling of external photon beams in radiotherapy," Monte Carlo Techniques in Radiation Therapy (CRC Press, 2013), Chap. 5, p. 63.

${ }^{100}$ M. Fragoso, I. Kawrakow, B. A. Faddegon, T. D. Solberg, and I. J. Chetty, "Fast, accurate photon beam accelerator modeling using BEAMnrc: A systematic investigation of efficiency enhancing methods and crosssection data," Med. Phys. 36, 5451-5466 (2009).

${ }^{101}$ L. Brualla, R. Palanco-Zamora, K.-P. Steuhl, N. Bornfeld, and W. Sauerwein, "Monte Carlo simulations applied to conjunctival lymphoma radiotherapy treatment," Strahlenther. Onkol. 187, 492-498 (2011).

${ }^{102}$ N. Chofor, D. Harder, K. Willborn, A. Rühmann, and B. Poppe, "Lowenergy photons in high-energy photon fields-Monte Carlo generated spectra and a new descriptive parameter," Z. Med. Phys. 21, 183-197 (2011).

${ }^{103}$ E. Sterpin, Y. Chen, Q. Chen, W. Lu, T. R. Mackie, and S. Vynckier, "Monte Carlo-based simulation of dynamic jaws tomotherapy," Med. Phys. 38, 5230-5238 (2011)

${ }^{104}$ M. K. Fix, W. Volken, D. Frei, D. Frauchiger, E. J. Born, and P. Manser, "Monte Carlo implementation, validation, and characterization of a 120 leaf MLC," Med. Phys. 38, 5311-5320 (2011).

${ }^{105}$ J. Belec, N. Ploquin, D. J. La Russa, and B. G. Clark, "Positionprobability-sampled Monte Carlo calculation of VMAT, 3DCRT, stepshoot IMRT, and helical tomotherapy dose distributions using BEAMnrc/DOSXYZnrc," Med. Phys. 38, 948-960 (2011).

${ }^{106}$ B. Vanderstraeten, N. Reynaert, L. Paelinck, I. Madani, C. De Wagter, W. De Gersem, W. De Neve, and H. Thierens, "Accuracy of patient dose calculation for lung IMRT: A comparison of Monte Carlo, convolution/superposition, and pencil beam computations," Med. Phys. 33, 31493158 (2006)

${ }^{107}$ L. Grevillot, D. Bertrand, F. Dessy, N. Freud, and D. Sarrut, "A Monte Carlo pencil beam scanning model for proton treatment plan simulation using GATE/GEANT4," Phys. Med. Biol. 56, 5203-5219 (2011).

${ }^{108}$ S. Benhalouche, S. Edel, D. Visvikis, O. Pradier, and N. Boussion, "Gate Monte Carlo simulation of a $6 \mathrm{MV}$ siemens oncor linear accelerator photon beam: Feasibility and preliminary dosimetric study," Med. Phys. 38, 3655 (2011).

${ }^{109}$ L. Grevillot, T. Frisson, N. Zahra, D. Bertrand, F. Stichelbaut, N. Freud, and D. Sarrut, "Optimization of GEANT4 settings for proton pencil beam scanning simulations using GATE,” Nucl. Instrum. Meth. Phys. Res. Sect. B 268, 3295-3305 (2010).

${ }^{110} \mathrm{H}$. Paganetti, "Four-dimensional Monte Carlo simulation of timedependent geometries,” Phys. Med. Biol. 49, N75 (2004).

${ }^{111}$ H. Paganetti, H. Jiang, S.-Y. Lee, and H. M. Kooy, "Accurate Monte Carlo simulations for nozzle design, commissioning and quality assurance for a proton radiation therapy facility," Med. Phys. 31, 2107-2118 (2004)

${ }^{112}$ H. Paganetti, H. Jiang, J. A. Adams, G. T. Y. Chen, and E. Rietzel, "Monte carlo simulations with time-dependent geometries to investigate effects of organ motion with high temporal resolution," Int. J. Radiat. Oncol., Biol., Phys. 60, 942-950 (2004).
${ }^{113}$ G. Cirrone, G. Cuttone, F. Di Rosa, S. Mazzaglia, F. Romano, A. Attili, F. Bourhaleb, G. Russo, P. Kataniemi, A. Heikkinen, and F. Marchetto, "Hadrontherapy: An open source, GEANT4-based application for protonion therapy studies," in Proceedings of the IEEE Nuclear Science Symposium Conference Record (NSS/MIC) (2009), p. 4186.

${ }^{114}$ M. Fippel, "Efficient particle transport simulation through beam modulating devices for Monte Carlo treatment planning," Med. Phys. 31, 12351242 (2004)

${ }^{115}$ M. Fippel and M. Soukup, "A Monte Carlo dose calculation algorithm for proton therapy," Med. Phys. 31, 2263-2273 (2004).

${ }^{116}$ M. Soukup, M. Fippel, and M. Alber, "A pencil beam algorithm for intensity modulated proton therapy derived from Monte Carlo simulations," Phys. Med. Biol. 50, 5089-5104 (2005).

${ }^{117}$ S. W. Peterson, J. Polf, M. Bues, G. Ciangaru, L. Archambault, S. Beddar, and A. Smith, "Experimental validation of a Monte Carlo proton therapy nozzle model incorporating magnetically steered protons," Phys. Med. Biol. 54, 3217-3229 (2009).

${ }^{118}$ T. Inaniwa, T. Furukawa, Y. Kase, N. Matsufuji, T. Toshito, Y. Matsumoto, Y. Furusawa, and K. Noda, "Treatment planning for a scanned carbon beam with a modified microdosimetric kinetic model," Phys. Med. Biol. 55, 6721-6737 (2010).

${ }^{119}$ Y. Jia, C. Beltran, D. J. Indelicato, S. Flampouri, Z. Li, and T. E. Merchant, "Proton therapy dose distribution comparison between Monte Carlo and a treatment planning system for pediatric patients with ependymoma," Med. Phys. 39, 4742-4747 (2012).

${ }^{120} \mathrm{~K}$. Parodi, W. Enghardt, and T. Haberer, "In-beam pet measurements of beta+ radioactivity induced by proton beams," Phys. Med. Biol. 47, 2136 (2002).

${ }^{121}$ S. Agostinelli, "GEANT4: A simulation toolkit," Nucl. Instrum. Meth. Phys. Res. Sect. A 506, 250-303 (2003).

${ }^{122}$ J. Allison, K. Amako, J. Apostolakis, H. Araujo, P. Arce Dubois, M. Asai, G. Barrand, R. Capra, S. Chauvie, R. Chytracek, G. Cirrone, G. Cooperman, G. Cosmo, G. Cuttone, G. Daquino, M. Donszelmann, M. Dressel, G. Folger, F. Foppiano, J. Generowicz, V. Grichine, S. Guatelli, P. Gumplinger, A. Heikkinen, I. Hrivnacova, A. Howard, S. Incerti, V. Ivanchenko, T. Johnson, F. Jones, T. Koi, R. Kokoulin, M. Kossov, H. Kurashige, V. Lara, S. Larsson, F. Lei, O. Link, F. Longo, M. Maire, A. Mantero, B. Mascialino, I. McLaren, P. Mendez Lorenzo, K. Minamimoto, K. Murakami, P. Nieminen, L. Pandola, S. Parlati, L. Peralta, J. Perl, A. Pfeiffer, M. Pia, A. Ribon, P. Rodrigues, G. Russo, S. Sadilov, G. Santin, T. Sasaki, D. Smith, N. Starkov, S. Tanaka, E. Tcherniaev, B. Tome, A. Trindade, P. Truscott, L. Urban, M. Verderi, A. Walkden, J. Wellisch, D. Williams, D. Wright, and H. Yoshida, "GEANT4 developments and applications," IEEE Trans. Nucl. Sci. 53, 270-278 (2006)

${ }^{123} \mathrm{~S}$. Jan, T. Frisson, and D. Sarrut, "Gate simulation of $12 \mathrm{C}$ hadrontherapy treatment combined with a PET imaging system for dose monitoring: A feasibility study," IEEE Trans. Nucl. Sci. 60, 423-429 (2013).

${ }^{124}$ C. Robert, N. Fourrier, D. Sarrut, S. Stute, P. Gueth, L. Grevillot, and I. Buvat, "PET-based dose delivery verification in proton therapy: A GATE based simulation study of five PET system designs in clinical conditions," Phys. Med. Biol. 58, 6867-6885 (2013).

${ }^{125}$ E. Seravalli, C. Robert, J. Bauer, F. Stichelbaut, C. Kurz, J. Smeets, C. Van Ngoc Ty, D. R. Schaart, I. Buvat, K. Parodi, and F. Verhaegen, "Monte Carlo calculations of positron emitter yields in proton radiotherapy," Phys. Med. Biol. 57, 1659-1673 (2012).

${ }^{126}$ I. Pshenichnov, I. Mishustin, and W. Greiner, "Distributions of positronemitting nuclei in proton and carbon-ion therapy studied with GEANT4," Phys. Med. Biol. 51, 6099-6112 (2006).

${ }^{127}$ S. España, X. Zhu, J. Daartz, G. El Fakhri, T. Bortfeld, and H. Paganetti, "The reliability of proton-nuclear interaction cross-section data to predict proton-induced pet images in proton therapy," Phys. Med. Biol. 56, 2687 2698 (2011)

${ }^{128}$ L. Lestand, G. Montarou, P. Force, and N. Pauna, "In-beam quality assurance using induced $\beta(+)$ activity in hadrontherapy: A preliminary physical requirements study using GEANT4,” Phys. Med. Biol. 57, 6497-6518 (2012).

${ }^{129}$ H. Mizuno, T. Tomitani, M. Kanazawa, A. Kitagawa, J. Pawelke, Y. Iseki, E. Urakabe, M. Suda, A. Kawano, R. Iritani, S. Matsushita, T. Inaniwa, T. Nishio, S. Furukawa, K. Ando, Y. K. Nakamura, T. Kanai, and K. Ishii, "Washout measurement of radioisotope implanted by radioactive beams in the rabbit," Phys. Med. Biol. 48, 2269-2281 (2003). 
${ }^{130}$ S. Surti, W. Zou, M. E. Daube-Witherspoon, J. McDonough, and J. S. Karp, "Design study of an in situ PET scanner for use in proton beam therapy," Phys. Med. Biol. 56, 2667-2685 (2011).

${ }^{131}$ J. C. Polf, S. Peterson, M. McCleskey, B. T. Roeder, A. Spiridon, S. Beddar, and L. Trache, "Measurement and calculation of characteristic prompt gamma ray spectra emitted during proton irradiation," Phys. Med. Biol. 54, N519-N527 (2009).

${ }^{132}$ P. Gueth, D. Dauvergne, N. Freud, J. M. Létang, C. Ray, E. Testa, and D. Sarrut, "Machine learning-based patient specific prompt-gamma dose monitoring in proton therapy," Phys. Med. Biol. 58, 4563-4577 (2013).

${ }^{133}$ F. Le Foulher, M. Bajard, M. Chevallier, D. Dauvergne, N. Freud, P. Henriquet, S. Karkar, J. M. Létang, L. Lestand, R. Plescak, C. Ray, D. Schardt, E. Testa, and M. Testa, "Monte Carlo simulations of prompt-gamma emission during carbon ion irradiation,” IEEE Trans. Nucl. Sci. 57, 2768-2772 (2010).

${ }^{134}$ Envision, ENVISION delivrable D3.1, Technical Report, 2012.

${ }^{135}$ M. Frandes, A. Zoglauer, V. Maxim, and R. Prost, “A tracking comptonscattering imaging system for hadron therapy monitoring," IEEE Trans. Nucl. Sci. 57, 144-150 (2010).

${ }^{136} \mathrm{~S}$. W. Peterson, D. Robertson, and J. Polf, “Optimizing a three-stage compton camera for measuring prompt gamma rays emitted during proton radiotherapy," Phys. Med. Biol. 55, 6841-6856 (2010).

${ }^{137}$ M.-H. Richard, D. Dauvergne, M. Dahoumane, N. Freud, P. Henriquet, J. M. Létang, J. Krimmer, C. Ray, E. Testa, and A. H. Walenta, "Design of a Compton camera for hadrontherapy on-line control using GEANT4," in Proceedings of the IEEE Nuclear Science Symposium and Medical Imaging Conference, 2011.
${ }^{138}$ V. Bom, L. Joulaeizadeh, and F. Beekman, "Real-time prompt gamma monitoring in spot-scanning proton therapy using imaging through a knife-edge-shaped slit," Phys. Med. Biol. 57, 297-308 (2012).

${ }^{139}$ P. Cambraia-Lopes, M. Pinto, H. Simões, A. Biegun, P. Dendooven, D. Oxley, K. Parodi, D. Schaart, and P. Crespo, "Optimization of collimator designs for real-time proton range verification by measuring prompt gamma rays," in Proceedings of the IEEE Nuclear Science Symposium and Medical Imaging Conference, 2012.

${ }^{140}$ A. K. Biegun, E. Seravalli, P. C. Lopes, I. Rinaldi, M. Pinto, D. C. Oxley, P. Dendooven, F. Verhaegen, K. Parodi, P. Crespo, and D. R. Schaart, "Time-of-flight neutron rejection to improve prompt gamma imaging for proton range verification: A simulation study," Phys. Med. Biol. 57, 6429_ 6444 (2012)

${ }^{141}$ M. Moteabbed, S. Espana, and H. Paganetti, "Monte Carlo patient study on the comparison of prompt gamma and pet imaging for range verification in proton therapy," Phys. Med. Biol. 56, 1063-1082 (2011).

${ }^{142}$ C. Robert, G. Dedes, G. Battistoni, T. T. Böhlen, I. Buvat, F. Cerutti, M. P. W. Chin, A. Ferrari, P. Gueth, C. Kurz, L. Lestand, A. Mairani, G. Montarou, R. Nicolini, P. G. Ortega, K. Parodi, Y. Prezado, P. R. Sala, D. Sarrut, and E. Testa, "Distributions of secondary particles in proton and carbon-ion therapy: A comparison between GATE/Geant4 and FLUKA Monte Carlo codes," Phys. Med. Biol. 58, 2879-2899 (2013).

${ }^{143}$ T. Bohlen, F. Cerutti, M. Dosanjh, A. Ferrari, I. Gudowska, A. Mairani, J. Quesada, and T. Böhlen, "Benchmarking nuclear models of FLUKA and GEANT4 for carbon ion therapy," Phys. Med. Biol. 55, 5833-5847 (2010) 